\title{
FINITE ELEMENT DISCRETIZATION OF THE STOKES AND NAVIER-STOKES EQUATIONS WITH BOUNDARY CONDITIONS ON THE PRESSURE*
}

\author{
CHRISTINE BERNARDI ${ }^{\dagger}$, TOMÁS CHACÓN REBOLLO ${ }^{\ddagger}$, AND DRISS YAKOUBI§
}

\begin{abstract}
We consider the Stokes and Navier-Stokes equations with boundary conditions of Dirichlet type on the velocity on one part of the boundary and involving the pressure on the rest of the boundary. We write the variational formulations of such problems. Next we propose a finite element discretization of them and perform the a priori and a posteriori analysis of the discrete problem. Some numerical experiments are presented in order to justify our strategy.
\end{abstract}

Key words. Navier-Stokes equations, new boundary conditions, finite elements

AMS subject classifications. 35Q30, 65N30, 76D05

DOI. $10.1137 / 140972299$

1. Introduction. Most works concerning the Stokes or Navier-Stokes equations deal with Dirichlet boundary conditions on the velocity (also called no-slip conditions); see, for instance, [19] or [32]. However, other types of boundary conditions were suggested in the pioneering paper [4], which was followed by a large number of works on this subject. Among them, the conditions on the normal component of the velocity and the vorticity were thoroughly studied and led to the so-called vorticity-velocitypressure formulation, introduced in [30] and studied in several other papers; see [16], [17], and [7], for instance; their extension to mixed boundary conditions was performed in [8]. However, it seems that the conditions on the tangential components of the velocity and the pressure have less been studied; we refer the reader to [28] and [14] for first works on these topics and also to [5] in the case of a simple geometry and of the linear Stokes problem. Recent papers deal either with the analysis of the equations [3], [25] or with their discretization [22], [23], [27], [31]. Unfortunately this discretization most often relies on finite difference schemes.

We wish here to propose a discretization in the case of mixed boundary conditions, Dirichlet conditions on the velocity in part of the boundary, conditions on the tangential components of the velocity and on the pressure on another part, both for the Stokes and Navier-Stokes equations. We first write the variational formulation of these problems and recall their main properties. It can be noted that all conditions on the velocity are prescribed in an essential way, while the boundary condition on the pressure is treated in a natural way. Next, we consider a finite element discretization: In view of the variational formulation, we decide to use the same finite elements as for standard boundary conditions, more precisely the Taylor-Hood element [21]. We

* Received by the editors June 9, 2014; accepted for publication (in revised form) March 4, 2015; published electronically May 14, 2015.

http://www.siam.org/journals/sinum/53-3/97229.html

${ }^{\dagger}$ Laboratoire Jacques-Louis Lions, C.N.R.S. and Université Pierre et Marie Curie, 75252 Paris Cedex 05, France (bernardi@ann.jussieu.fr).

${ }^{\ddagger}$ Departamento de Ecuaciones Diferenciales y Analisis Numrico \& Instituto de Matemática de la Universidad de Sevilla (IMUS),Universidad de Sevilla, 41012 Sevilla, Spain (chacon@us.es). The research of this author was partially funded by the Spanish Ministerio de Economía e Innovación and by FEDER EU grant MTM2012-36124-C02-01.

$\S$ GIREF, Département de Mathématiques et de Statistique, Pavillon Vachon, Université de Laval, Québec, QC G1V OA6, Canada (yakoubi@giref.ulaval.ca).

1256 
perform the numerical analysis of the discrete problem: Optimal a priori estimates and quasi-optimal a posteriori error estimates are derived, both in the linear and nonlinear cases. The arguments are similar to those for standard boundary conditions but require small extensions. In a final step, we present numerical experiments that justify the strategy of the discretization we choose.

The outline of this article is as follows:

- In section 2, we present the variational formulation of the full system and investigate its well-posedness.

- Section 3 is devoted to the description and a priori and a posteriori error analysis of the discretization of the Stokes problem.

- The a priori and a posteriori analysis of the discretization applied to the Navier-Stokes equations are the object of section 4.

- In section 5, we present some numerical experiments.

2. The continuous problem and its well-posedness. Let $\Omega$ be a bounded connected domain in $\mathbb{R}^{d}, d=2$ or 3 , with a Lipschitz-continuous and connected boundary $\partial \Omega$. We assume that this boundary admits a partition without overlap into two parts,

$$
\partial \Omega=\bar{\Gamma}_{1} \cup \bar{\Gamma}_{2}, \quad \Gamma_{1} \cap \Gamma_{2}=\emptyset,
$$

where both $\Gamma_{1}$ and $\Gamma_{2}$ have a finite number of connected components. From now on, we also assume that both $\Gamma_{1}$ and $\Gamma_{2}$ have a positive measure in $\partial \Omega$. We denote by $\boldsymbol{n}$ the unit vector normal to $\partial \Omega$ and exterior to $\Omega$.

From now on, we use the notation of the three-dimensional case and sometimes explain the modification in dimension $d=2$. Thus, we consider the following problems for $\varepsilon=0$ and $\varepsilon=1$ :

$$
\left\{\begin{aligned}
-\nu \Delta \boldsymbol{u}+\varepsilon(\boldsymbol{u} \cdot \nabla) \boldsymbol{u}+\operatorname{grad} p=\boldsymbol{f} & \text { in } \Omega, \\
\operatorname{div} \boldsymbol{u}=0 & \text { in } \Omega, \\
\boldsymbol{u}=\boldsymbol{u}_{1} & \text { on } \Gamma_{1}, \\
\boldsymbol{u} \times \boldsymbol{n}=\boldsymbol{u}_{2} \times \boldsymbol{n} & \text { on } \Gamma_{2}, \\
p+\frac{\varepsilon}{2}|\boldsymbol{u}|^{2}=p_{2} & \text { on } \Gamma_{2} .
\end{aligned}\right.
$$

(In dimension $d=2$, the third component of $\boldsymbol{n}$ is zero, so that $\boldsymbol{u} \times \boldsymbol{n}$ and $\boldsymbol{u}_{2} \times \boldsymbol{n}$ mean the tangential component of $\boldsymbol{u}$ and $\boldsymbol{u}_{2}$, respectively, each of which is scalar.) Indeed, the first two lines correspond to the standard Stokes model for $\varepsilon=0$ and to the Navier-Stokes equations for $\varepsilon=1$. The unknowns are the velocity $\boldsymbol{u}$ and the pressure $p$ of the fluid, while the quantity $p+\frac{1}{2}|\boldsymbol{u}|^{2}$ represents the dynamical pressure. The data are a density of forces $\boldsymbol{f}$ on the whole domain and the boundary data $\boldsymbol{u}_{1}$, $\boldsymbol{u}_{2}$, and $p_{2}$, while the viscosity $\nu$ is a positive constant.

We write a variational formulation of problem (2.1); next we prove the existence of a solution first for $\varepsilon=0$ and then for $\varepsilon=1$.

2.1. The variational formulation. With standard notation for the Sobolev spaces $H^{s}(\Omega)$ and $H_{0}^{s}(\Omega)$ (see [1, Chap. 3] for details), we introduce the domains of the divergence and curl operators,

$$
\begin{aligned}
H(\operatorname{div} ; \Omega) & =\left\{\boldsymbol{v} \in L^{2}(\Omega)^{d} ; \operatorname{div} \boldsymbol{v} \in L^{2}(\Omega)\right\} \\
H(\operatorname{curl} ; \Omega) & =\left\{\boldsymbol{v} \in L^{2}(\Omega)^{d} ; \operatorname{curl} \boldsymbol{v} \in L^{2}(\Omega)^{\frac{d(d-1)}{2}}\right\} .
\end{aligned}
$$

Copyright (c) by SIAM. Unauthorized reproduction of this article is prohibited. 
We recall from [19, Chap. I, sects. 2.2 and 2.3] that the normal trace operator, $\boldsymbol{v} \mapsto$ $\boldsymbol{v} \cdot \boldsymbol{n}$, is continuous from $H(\operatorname{div} ; \Omega)$ into $H^{-\frac{1}{2}}(\partial \Omega)$ and that the tangential trace operator, $\boldsymbol{v} \mapsto \boldsymbol{v} \times \boldsymbol{n}$, is continuous from $H(\operatorname{curl} ; \Omega)$ into $H^{-\frac{1}{2}}(\partial \Omega)^{\frac{d(d-1)}{2}}$. Thus we introduce our variational space

$$
\mathbb{X}=\left\{\boldsymbol{v} \in H(\operatorname{div} ; \Omega) \cap H(\operatorname{curl} ; \Omega) ; \boldsymbol{v} \cdot \boldsymbol{n}=\mathbf{0} \text { on } \Gamma_{1} \text { and } \boldsymbol{v} \times \boldsymbol{n}=\mathbf{0} \text { on } \partial \Omega\right\}
$$

Obviously, the trace operator $\boldsymbol{v} \rightarrow \boldsymbol{v} \cdot \boldsymbol{n}$ is continuous from $\mathbb{X}$ onto the dual space of $H_{00}^{\frac{1}{2}}\left(\Gamma_{2}\right)$ (see [24, Chap. 1, sect. 11.3] for the definition of the space $H_{00}^{\frac{1}{2}}\left(\Gamma_{2}\right)$ ). So, we denote by $H_{00}^{-\frac{1}{2}}\left(\Gamma_{2}\right)$ its dual space and by $\langle\cdot, \cdot\rangle_{\Gamma_{2}}$ the corresponding duality pairing.

Remark 2.1. Let $\Omega^{*}$ be any domain included in $\Omega$ such that $\partial \Omega^{*} \cap \partial \Omega$ is contained in $\Gamma_{1}$. The restrictions of functions of $\mathbb{X}$ to $\Omega^{*}$ belong to $H^{1}\left(\Omega^{*}\right)^{d}$; see [2, Thm. 2.5], for instance. On the other hand, when $\Gamma_{2}$ is of class $\mathcal{C}^{1,1}$ or convex (where "convex" means that there exists a convex neighbourhood of $\Gamma_{2}$ in $\Omega$ ), it can be proven $[2$, Thms. 2.12 and 2.17] that $\mathbb{X}$ is imbedded in $H^{1}(\Omega)^{d}$. Unfortunately, when $\Gamma_{2}$ has re-entrant corners or edges, it is only imbedded in $H^{\frac{1}{2}}(\Omega)^{d}$; see [15].

The aim of the space $\mathbb{X}$ is of course to take into account the boundary conditions on the velocity (we recall that, in dimension $d=2, \boldsymbol{v} \times \boldsymbol{n}=\mathbf{0}$ means that the tangential component of $\boldsymbol{v}$ vanishes). Next we define the bilinear forms

$$
a(\boldsymbol{u}, \boldsymbol{v})=\nu \int_{\Omega}(\operatorname{curl} \boldsymbol{u})(\boldsymbol{x}) \cdot(\operatorname{curl} \boldsymbol{v})(\boldsymbol{x}) d \boldsymbol{x}, \quad b(\boldsymbol{v}, q)=-\int_{\Omega}(\operatorname{div} \boldsymbol{v})(\boldsymbol{x}) q(\boldsymbol{x}) d \boldsymbol{x}
$$

together with the trilinear form

$$
N(\boldsymbol{w}, \boldsymbol{u}, \boldsymbol{v})=\int_{\Omega}(\operatorname{curl} \boldsymbol{u} \times \boldsymbol{w})(\boldsymbol{x}) \cdot \boldsymbol{v}(\boldsymbol{x}) d \boldsymbol{x}-\frac{1}{2} \int_{\Omega}(\boldsymbol{u} \cdot \boldsymbol{w})(\boldsymbol{x})(\operatorname{div} \boldsymbol{v})(\boldsymbol{x}) d \boldsymbol{x}
$$

Note that, in dimension $d=2, \operatorname{curl} \boldsymbol{u}$ is a scalar function, so that $\operatorname{curl} \boldsymbol{u} \times \boldsymbol{w}$ means the vector function with components $(\operatorname{curl} \boldsymbol{u}) w_{y}$ and $-(\operatorname{curl} \boldsymbol{u}) w_{x}$. With this notation, we consider the following problem: Find $(\boldsymbol{u}, p)$ in $(H(\operatorname{div} ; \Omega) \cap H(\operatorname{curl} ; \Omega)) \times L^{2}(\Omega)$ such that

$$
\begin{array}{r}
\boldsymbol{u}=\boldsymbol{u}_{1} \text { on } \Gamma_{1} \quad \text { and } \quad \boldsymbol{u} \times \boldsymbol{n}=\boldsymbol{u}_{2} \times \boldsymbol{n} \text { on } \Gamma_{2} \\
\forall \boldsymbol{v} \in \mathbb{X}, \quad a(\boldsymbol{u}, \boldsymbol{v})+\varepsilon N(\boldsymbol{u}, \boldsymbol{u}, \boldsymbol{v})+b(\boldsymbol{v}, p)=\int_{\Omega} \boldsymbol{f}(\boldsymbol{x}) \cdot \boldsymbol{v}(\boldsymbol{x}) d \boldsymbol{x}-\left\langle p_{2}, \boldsymbol{v} \cdot \boldsymbol{n}\right\rangle_{\Gamma_{2}} \\
\forall q \in L^{2}(\Omega), \quad b(\boldsymbol{u}, q)=0 .
\end{array}
$$

Indeed, we have the following result.

Proposition 2.2. Any solution $(\boldsymbol{u}, p)$ of the variational problem (2.5)-(2.6) such that $p$ belongs to $H^{1}(\Omega)$ is a solution of problem (2.1) (in the distribution sense). Conversely, any solution $(\boldsymbol{u}, p)$ of problem $(2.1)$ which belongs to $\mathcal{C}^{2}(\Omega)^{d} \times \mathcal{C}^{1}(\Omega)$ and also to $\mathcal{C}^{0}(\bar{\Omega})^{d} \times \mathcal{C}^{0}(\bar{\Omega})$ is a solution of the variational problem $(2.5)-(2.6)$.

Proof. The third and fourth lines in (2.1) are obviously equivalent to (2.5). On the other hand, taking $q$ equal to $\operatorname{div} \boldsymbol{u}$ in (2.6) yields the second line in (2.1). Finally, we recall that, by integration by parts and for a function $\boldsymbol{v}$ in $\mathcal{D}(\bar{\Omega})^{d} \cap \mathbb{X}$ (note that such a function has its trace $\boldsymbol{v} \times \boldsymbol{n}$ equal to zero on all the boundary $\partial \Omega$ and that a 
weak regularity of $p$ is needed for the last line),

$$
\begin{aligned}
a(\boldsymbol{u}, \boldsymbol{v}) & =\nu \int_{\Omega} \operatorname{curl}(\operatorname{curl} \boldsymbol{u})(\boldsymbol{x}) \cdot \boldsymbol{v}(\boldsymbol{x}) d \boldsymbol{x}, \\
N(\boldsymbol{u}, \boldsymbol{u}, \boldsymbol{v}) & =\int_{\Omega}((\boldsymbol{u} \cdot \nabla) \boldsymbol{u})(\boldsymbol{x}) \cdot \boldsymbol{v}(\boldsymbol{x}) d \boldsymbol{x}-\frac{1}{2} \int_{\Gamma_{2}}|\boldsymbol{u}|^{2}(\tau)(\boldsymbol{v} \cdot \boldsymbol{n})(\tau) d \tau, \\
b(\boldsymbol{v}, p) & =\int_{\Omega} \boldsymbol{v}(\boldsymbol{x}) \cdot \operatorname{grad} p(\boldsymbol{x}) d \boldsymbol{x}-\langle p, \boldsymbol{v} \cdot \boldsymbol{n}\rangle_{\Gamma_{2}},
\end{aligned}
$$

where $\tau$ stands for the tangential coordinate(s) on $\partial \Omega$. Then, thanks to the identity

$$
-\Delta \boldsymbol{u}=\operatorname{curl}(\operatorname{curl} \boldsymbol{u})-\operatorname{grad}(\operatorname{div} \boldsymbol{u}),
$$

taking $\boldsymbol{v}$ in $\mathcal{D}(\Omega)^{d}$ gives the first equation in (2.1). The fifth equation then follows by taking $\boldsymbol{v}$ in $\mathcal{D}(\bar{\Omega})^{d} \cap \mathbb{X}$ and looking at the terms on $\Gamma_{2}$ issued from (2.6).

The converse property is proved by the same arguments, together with the regularity of $(\boldsymbol{u}, p)$.

We now prove the existence of a solution for problem (2.5)-(2.6).

2.2. The Stokes problem. In the case $\varepsilon=0$ of the Stokes problem, problem $(2.5)-(2.6)$ is of standard saddle-point type. So, its well-posedness requires two infsup conditions. The first one is an extension of the usual inf-sup condition for the Stokes problem to our boundary conditions; its proof can be found in [5, Proof of Thm. 2.1] or [6, Lem. 3.1]. The space $\mathbb{X}$ is now provided with the graph norm of $H(\operatorname{div} ; \Omega) \cap H(\operatorname{curl} ; \Omega)$, i.e.,

$$
\|\boldsymbol{v}\|_{\mathbb{X}}=\left(\|\boldsymbol{v}\|_{L^{2}(\Omega)^{d}}^{2}+\|\operatorname{div} \boldsymbol{v}\|_{L^{2}(\Omega)}^{2}+\|\operatorname{curl} \boldsymbol{v}\|_{L^{2}(\Omega)}^{2} \frac{d(d-1)}{2}\right)^{\frac{1}{2}},
$$

which is smaller than $\|\cdot\|_{H^{1}(\Omega)^{d}}$.

LEMma 2.3. There exists a constant $\beta>0$ such that the following inf-sup condition holds:

$$
\forall q \in L^{2}(\Omega), \quad \sup _{\boldsymbol{v} \in \mathbb{X}} \frac{b(\boldsymbol{v}, q)}{\|\boldsymbol{v}\|_{\mathbb{X}}} \geq \beta\|q\|_{L^{2}(\Omega)} .
$$

The next lemma requires the kernel

$$
\mathbb{V}=\left\{\boldsymbol{v} \in \mathbb{X} ; \forall q \in L^{2}(\Omega), b(\boldsymbol{v}, q)=0\right\},
$$

which is obviously characterized by

$$
\mathbb{V}=\{\boldsymbol{v} \in \mathbb{X} ; \operatorname{div} \boldsymbol{v}=0 \text { in } \Omega\} .
$$

LEMma 2.4. There exists a constant $\alpha>0$ such that the following ellipticity property holds:

$$
\forall \boldsymbol{v} \in \mathbb{V}, \quad a(\boldsymbol{v}, \boldsymbol{v}) \geq \alpha\|\boldsymbol{v}\|_{\mathbb{X}}^{2} .
$$

Proof. Due to the definition of $\mathbb{V}$, we have for all $\boldsymbol{v}$ in $\mathbb{V}$ that

$$
a(\boldsymbol{v}, \boldsymbol{v})=\nu\left(\|\operatorname{curl} \boldsymbol{v}\|_{L^{2}(\Omega)}^{2} \frac{d(d-1)}{2}+\|\operatorname{div} \boldsymbol{v}\|_{L^{2}(\Omega)}^{2}\right) .
$$

Copyright $@$ by SIAM. Unauthorized reproduction of this article is prohibited. 
Since the boundary of $\Omega$ is connected, this last quantity is bounded from below by $c\|\boldsymbol{v}\|_{\mathbb{X}}^{2}$ (see [2, Cor. 3.19]), whence the desired ellipticity property.

We are now in a position to prove the first existence result. For any data $\boldsymbol{u}_{1}$ on $\Gamma_{1}$ and $\boldsymbol{u}_{2}$ on $\Gamma_{2}$, we denote by $\mathcal{C}\left(\boldsymbol{u}_{1}, \boldsymbol{u}_{2}\right)$ the function equal to $\boldsymbol{u}_{1}$ on $\Gamma_{1}$ and to $\boldsymbol{u}_{2}$ on $\Gamma_{2}$.

TheOrem 2.5. Assume that the data $\boldsymbol{f}, \boldsymbol{u}_{1}, \boldsymbol{u}_{2}$, and $p_{2}$ satisfy

$$
\boldsymbol{f} \in L^{2}(\Omega)^{d}, \quad \mathcal{C}\left(\boldsymbol{u}_{1}, \boldsymbol{u}_{2}\right) \in H^{\frac{1}{2}}(\partial \Omega)^{d}, \quad p_{2} \in H_{00}^{\frac{1}{2}}\left(\Gamma_{2}\right) .
$$

Then, problem (2.5)-(2.6) for $\varepsilon=0$ has a unique solution $(\boldsymbol{u}, p)$. Moreover, this solution satisfies

$$
\|\boldsymbol{u}\|_{\mathbb{X}}+\|p\|_{L^{2}(\Omega)} \leq c\left(\|\boldsymbol{f}\|_{L^{2}(\Omega)^{d}}+\left\|\mathcal{C}\left(\boldsymbol{u}_{1}, \boldsymbol{u}_{2}\right)\right\|_{H^{\frac{1}{2}(\partial \Omega)^{d}}}+\left\|p_{2}\right\|_{H_{00}^{\frac{1}{2}}\left(\Gamma_{2}\right)}\right) .
$$

Proof. Let $\boldsymbol{w}$ be a function in $H^{1}(\Omega)^{d}$ such that its trace on $\partial \Omega$ coincides with $\mathcal{C}\left(\boldsymbol{u}_{1}, \boldsymbol{u}_{2}\right)$ and which, moreover, satisfies

$$
\|\boldsymbol{w}\|_{H^{1}(\Omega)^{d}} \leq c\left\|\mathcal{C}\left(\boldsymbol{u}_{1}, \boldsymbol{u}_{2}\right)\right\|_{H^{\frac{1}{2}}(\partial \Omega)^{d}} .
$$

Then, the pair $\left(\boldsymbol{u}_{0}, p\right)$, with $\boldsymbol{u}_{0}=\boldsymbol{u}-\boldsymbol{w}$, must be found in $\mathbb{X} \times L^{2}(\Omega)$ and satisfy

$$
\begin{array}{r}
\forall \boldsymbol{v} \in \mathbb{X}, \quad a\left(\boldsymbol{u}_{0}, \boldsymbol{v}\right)+b(\boldsymbol{v}, p)=\int_{\Omega} \boldsymbol{f}(\boldsymbol{x}) \cdot \boldsymbol{v}(\boldsymbol{x}) d \boldsymbol{x}-\left\langle p_{2}, \boldsymbol{v} \cdot \boldsymbol{n}\right\rangle_{\Gamma_{2}}-a(\boldsymbol{w}, \boldsymbol{v}), \\
\forall q \in L^{2}(\Omega), \quad b\left(\boldsymbol{u}_{0}, q\right)=-b(\boldsymbol{w}, q) .
\end{array}
$$

The well-posedness of this last problem follows from Lemmas 2.3 and 2.4; see [19, Chap. I, Cor. 4.1]. This yields the existence and uniqueness of a solution to problem (2.5)-(2.6), together with estimate (2.12).

Remark 2.6. All this study makes use of data $p_{2}$ in $H_{00}^{\frac{1}{2}}\left(\Gamma_{2}\right)$ for generality. However, it follows from [15] that $p_{2}$ can often be less regular, for instance in $L^{2}\left(\Gamma_{2}\right)$ when $\Omega$ is a polygon or a polyhedron.

2.3. The Navier-Stokes equations. In the case $\varepsilon=1$ of the Navier-Stokes equations, we decide to work with homogeneous boundary conditions on the velocity, namely

$$
\boldsymbol{u}=\mathbf{0} \text { on } \Gamma_{1} \quad \text { and } \quad \boldsymbol{u} \times \boldsymbol{n}=\mathbf{0} \text { on } \Gamma_{2},
$$

in order to avoid the technical difficulties due to the Hopf lemma; see [19, Chap. IV, Lem. 2.3], for instance. Proving the existence of a solution relies on Brouwer's fixed point theorem and requires the next lemma.

Lemma 2.7. The spaces $\mathbb{X}$ and $\mathbb{V}$ are separable.

Proof. The space $\mathcal{D}(\bar{\Omega})^{d}$ is dense in $H(\operatorname{div} ; \Omega) \cap H(\operatorname{curl} ; \Omega)$ (see [2, Prop. 2.3]), so this space is separable. Since it is a Banach space and $\mathbb{X}$ is a closed subspace of it (this is due to the continuity of the trace), $\mathbb{X}$ is also separable; see [11, Prop. 3.22], for instance. Finally, since $\mathbb{V}$ is a closed subspace of $\mathbb{X}$, it is once more separable.

The main result of this section requires a further assumption.

Assumption 2.8. The space $\mathbb{X}$ is compactly imbedded in $L^{4}(\Omega)^{d}$.

It follows from Remark 2.1 that this assumption always holds when $\Gamma_{2}$ is of class $\mathcal{C}^{1,1}$ or convex, and also from [15] that it holds when $\Omega$ is a two-dimensional polygon. 
However, Assumption 2.8 seems to be less restrictive as far as the geometry of the domain is concerned.

TheOrem 2.9. Assume that the data $\boldsymbol{f}$ and $p_{2}$ satisfy

$$
\boldsymbol{f} \in L^{2}(\Omega)^{d}, \quad p_{2} \in H_{00}^{\frac{1}{2}}\left(\Gamma_{2}\right) .
$$

Then, if Assumption 2.8 holds, problem (2.6)-(2.14) for $\varepsilon=1$ has at least a solution $(\boldsymbol{u}, p)$. Moreover, this solution satisfies

$$
\begin{array}{r}
\|\boldsymbol{u}\|_{\mathbb{X}} \leq \frac{c}{\nu}\left(\|\boldsymbol{f}\|_{L^{2}(\Omega)^{d}}+\left\|p_{2}\right\|_{H_{00}^{\frac{1}{2}\left(\Gamma_{2}\right)}}\right), \\
\|p\|_{L^{2}(\Omega)} \leq c\left(\|\boldsymbol{f}\|_{L^{2}(\Omega)^{d}}+\left\|p_{2}\right\|_{H_{00}^{\frac{1}{2}}\left(\Gamma_{2}\right)}\right)+\frac{c^{\prime}}{\nu^{2}}\left(\|\boldsymbol{f}\|_{L^{2}(\Omega)^{d}}+\left\|p_{2}\right\|_{H_{00}^{\frac{1}{2}\left(\Gamma_{2}\right)}}\right)^{2},
\end{array}
$$

where both constants $c$ and $c^{\prime}$ are independent of $\nu$.

Proof. We proceed in several steps.

Step 1. We first note that, if $(\boldsymbol{u}, p)$ is a solution of problem (2.6)-(2.14), its part $\boldsymbol{u}$ belongs to $\mathbb{V}$ and satisfies

$$
\forall \boldsymbol{v} \in \mathbb{V}, \quad a(\boldsymbol{u}, \boldsymbol{v})+\tilde{N}(\boldsymbol{u}, \boldsymbol{u}, \boldsymbol{v})=\int_{\Omega} \boldsymbol{f}(\boldsymbol{x}) \cdot \boldsymbol{v}(\boldsymbol{x}) d \boldsymbol{x}-\left\langle p_{2}, \boldsymbol{v} \cdot \boldsymbol{n}\right\rangle_{\Gamma_{2}},
$$

where the new trilinear form $\tilde{N}(\cdot, \cdot, \cdot)$ is defined by

$$
\tilde{N}(\boldsymbol{w}, \boldsymbol{u}, \boldsymbol{v})=\int_{\Omega}(\operatorname{curl} \boldsymbol{u} \times \boldsymbol{w})(\boldsymbol{x}) \cdot \boldsymbol{v}(\boldsymbol{x}) d \boldsymbol{x} .
$$

We first investigate the existence of a solution for this problem.

Step 2. Let us introduce the mapping $\Phi$, defined from $\mathbb{V}$ into its dual space by

$$
\langle\Phi(\boldsymbol{u}), \boldsymbol{v}\rangle=a(\boldsymbol{u}, \boldsymbol{v})+\tilde{N}(\boldsymbol{u}, \boldsymbol{u}, \boldsymbol{v})-\int_{\Omega} \boldsymbol{f}(\boldsymbol{x}) \cdot \boldsymbol{v}(\boldsymbol{x}) d \boldsymbol{x}+\left\langle p_{2}, \boldsymbol{v} \cdot \boldsymbol{n}\right\rangle_{\Gamma_{2}} .
$$

By noting that $\tilde{N}(\boldsymbol{u}, \boldsymbol{u}, \boldsymbol{u})$ is zero, we derive by the same arguments as in Lemma 2.4

$$
\langle\Phi(\boldsymbol{u}), \boldsymbol{u}\rangle \geq \alpha\|\boldsymbol{u}\|_{\mathbb{X}}^{2}-c\left(\boldsymbol{f}, p_{2}\right)\|\boldsymbol{u}\|_{\mathbb{X}}
$$

where the constant $c\left(\boldsymbol{f}, p_{2}\right)=\|\boldsymbol{f}\|_{L^{2}(\Omega)^{d}}+\left\|p_{2}\right\|_{H_{00}^{\frac{1}{2}\left(\Gamma_{2}\right)}}$ depends only on the data. Thus, $\langle\Phi(\boldsymbol{u}), \boldsymbol{u}\rangle$ is nonnegative on the sphere with radius $\frac{c\left(\boldsymbol{f}, p_{2}\right)}{\alpha}$ (note that $\alpha=c \nu$ ).

Step 3. It follows from Lemma 2.7 that there exists an increasing sequence of finite-dimensional subspaces $\mathbb{V}_{n}$ of $\mathbb{V}$ such that $\cup_{n} \mathbb{V}_{n}$ is dense in $\mathbb{V}$. For any fixed $n$, the function $\Phi$ satisfies the same properties as previously on $\mathbb{V}_{n}$. So applying Brouwer's fixed point theorem (see [19, Chap. IV, Cor. 1.1], for instance) yields that there exists a $\boldsymbol{u}_{n}$ in $\mathbb{V}_{n}$ which satisfies

$$
\forall \boldsymbol{v}_{n} \in \mathbb{V}_{n}, \quad\left\langle\Phi\left(\boldsymbol{u}_{n}\right), \boldsymbol{v}_{n}\right\rangle=0
$$

Moreover, this $\boldsymbol{u}_{n}$ belongs to the ball with radius $\frac{c\left(\boldsymbol{f}, p_{2}\right)}{\alpha}$.

Step 4. Since the sequence $\left(\boldsymbol{u}_{n}\right)_{n}$ is bounded in $\mathbb{X}$, Assumption 2.8 implies that there exists a subsequence, still denoted by $\left(\boldsymbol{u}_{n}\right)_{n}$ for simplicity, which converges to a function $\boldsymbol{u}$ of $\mathbb{V}$ weakly in $\mathbb{X}$ and strongly in $L^{4}(\Omega)^{d}$. Moreover, due to the weak lower 
semicontinuity of the norm, the limit $\boldsymbol{u}$ still belongs to the ball with radius $\frac{c\left(\boldsymbol{f}, p_{2}\right)}{\alpha}$ and hence satisfies the first part of estimate (2.16).

Step 5 . For a fixed $m \leq n$, since the sequence $\left(\mathbb{V}_{n}\right)_{n}$ is increasing, each function $\boldsymbol{u}_{n}$ satisfies

$$
\forall \boldsymbol{v}_{m} \in \mathbb{V}_{m}, \quad\left\langle\Phi\left(\boldsymbol{u}_{n}\right), \boldsymbol{v}_{m}\right\rangle=0 .
$$

Then, passing to the limit on $n$ follows from the previous convergence properties. Due to the density of $\cup_{m} \mathbb{V}_{m}$ into $\mathbb{V}$, it is thus readily checked that the function $\boldsymbol{u}$ satisfies

$$
\forall \boldsymbol{v} \in \mathbb{V}, \quad\langle\Phi(\boldsymbol{u}), \boldsymbol{v}\rangle=0,
$$

and hence is a solution of problem (2.17).

Step 6 . From the previous lines and thanks to the definition of $\mathbb{V}$, the quantity

$$
\int_{\Omega} \boldsymbol{f}(\boldsymbol{x}) \cdot \boldsymbol{v}(\boldsymbol{x}) d \boldsymbol{x}-\left\langle p_{2}, \boldsymbol{v} \cdot \boldsymbol{n}\right\rangle_{\Gamma_{2}}-a(\boldsymbol{u}, \boldsymbol{v})-N(\boldsymbol{u}, \boldsymbol{u}, \boldsymbol{v})
$$

vanishes for all $\boldsymbol{v}$ in $\mathbb{V}$. So, it follows from Lemma 2.3 (see [19, Chap. I, Lem. 4.1]) that there exists a $p$ in $L^{2}(\Omega)$ such that

$$
\forall \boldsymbol{v} \in \mathbb{X}, \quad b(\boldsymbol{v}, p)=\int_{\Omega} \boldsymbol{f}(\boldsymbol{x}) \cdot \boldsymbol{v}(\boldsymbol{x}) d \boldsymbol{x}-\left\langle p_{2}, \boldsymbol{v} \cdot \boldsymbol{n}\right\rangle_{\Gamma_{2}}-a(\boldsymbol{u}, \boldsymbol{v})-N(\boldsymbol{u}, \boldsymbol{u}, \boldsymbol{v}) .
$$

Thus, the pair $(\boldsymbol{u}, p)$ is a solution of problem (2.6)-(2.14).

Step 7 . It also follows from Lemma 2.3 that

$$
\|p\|_{L^{2}(\Omega)} \leq \beta^{-1} \sup _{\boldsymbol{v} \in \mathbb{X}} \frac{\int_{\Omega} \boldsymbol{f}(\boldsymbol{x}) \cdot \boldsymbol{v}(\boldsymbol{x}) d \boldsymbol{x}-\left\langle p_{2}, \boldsymbol{v} \cdot \boldsymbol{n}\right\rangle_{\Gamma_{2}}-a(\boldsymbol{u}, \boldsymbol{v})-N(\boldsymbol{u}, \boldsymbol{u}, \boldsymbol{v})}{\|\boldsymbol{v}\|_{\mathbb{X}}} .
$$

Thanks to the estimate on $\boldsymbol{u}$, the quantity $p$ satisfies the second part of (2.16).

It is readily checked that any solution $(\boldsymbol{u}, p)$ of problem (2.6)-(2.14) satisfies estimate (2.16). This yields the uniqueness of the solution, but unfortunately with a rather restrictive condition on the data.

TheOREM 2.10. Assume that the data $\boldsymbol{f}$ and $p_{2}$ satisfy (2.15) and, moreover,

$$
\frac{\|\boldsymbol{f}\|_{L^{2}(\Omega)^{d}}+\left\|p_{2}\right\|_{H_{00}^{\frac{1}{2}}\left(\Gamma_{2}\right)}}{\nu^{2}} \leq c
$$

for an appropriate constant $c$. Then, if Assumption 2.8 holds, problem (2.6)-(2.14) for $\varepsilon=1$ has at most a solution $(\boldsymbol{u}, p)$.

Proof. Let $\left(\boldsymbol{u}_{1}, p_{1}\right)$ and $\left(\boldsymbol{u}_{2}, p_{2}\right)$ be two solutions of (2.6)-(2.14). Then, $\boldsymbol{u}_{1}$ and $\boldsymbol{u}_{2}$ belong to $\mathbb{V}$, and their difference satisfies

$$
\forall \boldsymbol{v} \in \mathbb{V}, \quad a\left(\boldsymbol{u}_{1}-\boldsymbol{u}_{2}, \boldsymbol{v}\right)=\tilde{N}\left(\boldsymbol{u}_{2}, \boldsymbol{u}_{2}, \boldsymbol{v}\right)-\tilde{N}\left(\boldsymbol{u}_{1}, \boldsymbol{u}_{1}, \boldsymbol{v}\right) .
$$

Next, taking $\boldsymbol{v}$ equal to $\boldsymbol{u}_{1}-\boldsymbol{u}_{2}$ and noting that $\tilde{N}(\boldsymbol{w}, \boldsymbol{v}, \boldsymbol{v})$ vanishes for all $\boldsymbol{v}$, we obtain

$$
\nu\left\|\operatorname{curl}\left(\boldsymbol{u}_{1}-\boldsymbol{u}_{2}\right)\right\|_{L^{2}(\Omega) \frac{d(d-1)}{2}}^{2}=\tilde{N}\left(\boldsymbol{u}_{2}-\boldsymbol{u}_{1}, \boldsymbol{u}_{2}, \boldsymbol{u}_{1}-\boldsymbol{u}_{2}\right) .
$$

We recall that

$$
\forall \boldsymbol{w} \in \mathbb{V}, \quad\|\boldsymbol{w}\|_{\mathbb{X}} \leq c\|\operatorname{curl} \boldsymbol{w}\|_{L^{2}(\Omega)^{\frac{d(d-1)}{2}}}^{2},
$$

Copyright $\odot$ by SIAM. Unauthorized reproduction of this article is prohibited. 
so that using estimate (2.16) for $\boldsymbol{u}_{2}$ yields

$\nu\left\|\operatorname{curl}\left(\boldsymbol{u}_{1}-\boldsymbol{u}_{2}\right)\right\|_{L^{2}(\Omega)}^{2} \frac{d(d-1)}{2} \leq \frac{c}{\nu}\left(\|\boldsymbol{f}\|_{L^{2}(\Omega)^{d}}+\left\|p_{2}\right\|_{H_{00}^{\frac{1}{2}\left(\Gamma_{2}\right)}}\right)\left\|\operatorname{curl}\left(\boldsymbol{u}_{1}-\boldsymbol{u}_{2}\right)\right\|_{L^{2}(\Omega)}^{2} \frac{d(d-1)}{2}$.

Thus, when (2.18) is satisfied with a small enough constant $c, \operatorname{curl}\left(\boldsymbol{u}_{1}-\boldsymbol{u}_{2}\right)$ vanishes. It thus follows from [2, Cor. 3.19] that, since both $\boldsymbol{u}_{1}$ and $\boldsymbol{u}_{2}$ are divergence-free, they coincide.

In this case, the functions $p_{1}$ and $p_{2}$ satisfy

$$
\forall \boldsymbol{v} \in \mathbb{X}, \quad b\left(\boldsymbol{v}, p_{1}-p_{2}\right)=0,
$$

so that, owing to Lemma 2.3, they coincide. This concludes the proof.

2.4. A final remark. We consider once more problem (2.5)-(2.6) or (2.6)-(2.14) but now with the form $a(\cdot, \cdot)$ replaced by

$$
a_{\lambda}(\boldsymbol{u}, \boldsymbol{v})=\nu \int_{\Omega}((\operatorname{curl} \boldsymbol{u})(\boldsymbol{x}) \cdot \operatorname{curl} \boldsymbol{v}(\boldsymbol{x})+\lambda \operatorname{div} \boldsymbol{u}(\boldsymbol{x}) \operatorname{div} \boldsymbol{v}(\boldsymbol{x})) d \boldsymbol{x} .
$$

It is easy to check that, for a positive parameter $\lambda$, this modification does not change the problems at all and that all the previous results are still valid with the modified problems.

The main difference between the forms $a(\cdot, \cdot)$ and $a_{\lambda}(\cdot, \cdot)$ is that this new form satisfies the next stronger ellipticity property. The interest of this new property for the discretization is obvious: It leads to the stabilization of the divergence term.

Lemma 2.11. For any positive parameter $\lambda$ there exists a constant $\alpha>0$ such that the following ellipticity property holds:

$$
\forall \boldsymbol{v} \in \mathbb{X}, \quad a_{\lambda}(\boldsymbol{v}, \boldsymbol{v}) \geq \alpha \min \{1, \lambda\}\|\boldsymbol{v}\|_{\mathbb{X}}^{2} .
$$

3. Discretization of the Stokes problem. From now on, we assume that $\Omega$ is a polygon or a polyhedron. We introduce a regular family of triangulations of $\Omega$ (by triangles or tetrahedra), in the usual sense that, for each $h$, the following hold:

- $\bar{\Omega}$ is the union of all elements of $\mathcal{T}_{h}$.

- The intersection of two different elements of $\mathcal{T}_{h}$, if not empty, is a vertex or a whole edge or a whole face of both of them.

- The ratio of the diameter $h_{K}$ of any element $K$ of $\mathcal{T}_{h}$ to the diameter of its inscribed circle or sphere is smaller than a constant independent of $h$. As usual, $h$ stands for the maximum of the diameters $h_{K}$. We make the further nonrestrictive assumption that $\bar{\Gamma}_{1}$ and $\bar{\Gamma}_{2}$ are the union of whole edges $(d=2)$ or faces $(d=3)$ of elements of $\mathcal{T}_{h}$. From now on, $c, c^{\prime}, \ldots$ stand for generic constants that can vary from line to line but are always independent of $h$.

\subsection{The discrete problem and its well-posedness. Setting}

$$
\mathbb{Y}_{h}=\left\{v_{h} \in H^{1}(\Omega) ; \forall K \in \mathcal{T}_{h},\left.v_{h}\right|_{K} \in \mathcal{P}_{2}(K)\right\}
$$

we define the space of discrete velocities

$$
\mathbb{X}_{h}=\mathbb{Y}_{h}^{d} \cap \mathbb{X}
$$

Copyright (c) by SIAM. Unauthorized reproduction of this article is prohibited. 
and the space of discrete pressures

$$
\mathbb{M}_{h}=\left\{q_{h} \in H^{1}(\Omega) ; \forall K \in \mathcal{T}_{h},\left.q_{h}\right|_{K} \in \mathcal{P}_{1}(K)\right\} .
$$

Even if the following analysis is valid for general mixed finite elements, we have chosen this one, called the Taylor-Hood element (see [21]), which is widely used in the case of standard boundary conditions; see [19, Chap. II, sect. 4.2] for its main properties. We denote by $\mathcal{I}_{h}$ the standard Lagrange interpolation operator with values in $\mathbb{Y}_{h}$.

In view of Lemma 2.11, we have decided to work with $\lambda=1$, i.e., with the form $a_{1}(\cdot, \cdot)$. The discrete problem is then constructed by the Galerkin method; it reads: Find $\left(\boldsymbol{u}_{h}, p_{h}\right)$ in $\mathbb{Y}_{h}^{d} \times \mathbb{M}_{h}$ such that

$$
\begin{array}{r}
\boldsymbol{u}_{h}=\mathcal{I}_{h} \boldsymbol{u}_{1} \text { on } \Gamma_{1} \quad \text { and } \quad \boldsymbol{u}_{h} \times \boldsymbol{n}=\mathcal{I}_{h} \boldsymbol{u}_{2} \times \boldsymbol{n} \text { on } \Gamma_{2}, \\
\forall \boldsymbol{v}_{h} \in \mathbb{X}_{h}, \quad a_{1}\left(\boldsymbol{u}_{h}, \boldsymbol{v}_{h}\right)+b\left(\boldsymbol{v}_{h}, p_{h}\right)=\int_{\Omega} \boldsymbol{f}(\boldsymbol{x}) \cdot \boldsymbol{v}_{h}(\boldsymbol{x}) d \boldsymbol{x}-\left\langle p_{2}, \boldsymbol{v}_{h} \cdot \boldsymbol{n}\right\rangle_{\Gamma_{2}}, \\
\forall q_{h} \in \mathbb{M}_{h}, \quad b\left(\boldsymbol{u}_{h}, q_{h}\right)=0 .
\end{array}
$$

Proving its well-posedness relies on the same arguments as for the continuous problem; however, a further assumption is required for the first inf-sup condition.

Assumption 3.1. At most an edge $(d=2)$ or a face $(d=3)$ of an element of $\mathcal{T}_{h}$ is contained in $\Gamma_{2}$.

This assumption is not restrictive at all since it is always true for $h$ small enough and leads to the following lemma.

Lemma 3.2. If Assumption 3.1 holds, there exists a constant $\beta_{*}>0$ such that the following inf-sup condition holds:

$$
\forall q_{h} \in \mathbb{M}_{h}, \quad \sup _{\boldsymbol{v}_{h} \in \mathbb{X}_{h}} \frac{b\left(\boldsymbol{v}_{h}, q_{h}\right)}{\left\|\boldsymbol{v}_{h}\right\|_{\mathbb{X}}} \geq \beta_{*}\left\|q_{h}\right\|_{L^{2}(\Omega)} .
$$

Proof. For any $q_{h}$ in $\mathbb{M}_{h}$, we use the expansion

$$
q_{h}=\tilde{q}+\bar{q}, \quad \text { with } \quad \bar{q}=\frac{1}{\operatorname{meas}(\Omega)} \int_{\Omega} q_{h}(\boldsymbol{x}) d \boldsymbol{x} .
$$

Next, we proceed in three steps.

Step 1 . Since $\tilde{q}$ has a null integral on $\Omega$, the standard inf-sup condition (see $[19$, Chap. II, Thm. 4.2], for instance) implies that there exists a function $\tilde{\boldsymbol{v}}$ in $\mathbb{Y}_{h}^{d} \cap H_{0}^{1}(\Omega)^{d}$, hence in $\mathbb{X}_{h}$, such that

$$
\operatorname{div} \tilde{\boldsymbol{v}}=-\tilde{q} \quad \text { and } \quad\|\tilde{\boldsymbol{v}}\|_{\mathbb{X}} \leq c\|\tilde{q}\|_{L^{2}(\Omega)} .
$$

Step 2. Since $\bar{q}$ is a constant, we observe that, for any $\boldsymbol{v}$ in $\mathbb{X}$,

$$
b(\boldsymbol{v}, \bar{q})=-\bar{q} \int_{\Gamma_{2}}(\boldsymbol{v} \cdot \boldsymbol{n})(s) d s .
$$

We introduce a function $\varphi$ in $\mathcal{D}\left(\Omega \cup \Gamma_{2}\right)$ such that $\int_{\Gamma_{2}} \varphi(s) d s$ is a positive constant $c_{0}$. And we note that

$$
\int_{\Gamma_{2}} \mathcal{I}_{h} \varphi(s) d s \geq \int_{\Gamma_{2}} \varphi(s) d s-\left\|\varphi-\mathcal{I}_{h} \varphi\right\|_{L^{1}\left(\Gamma_{2}\right)} \geq c_{0}-c h^{2}
$$

Copyright $@$ by SIAM. Unauthorized reproduction of this article is prohibited. 
so that this integral is larger than $\frac{c_{0}}{2}$ for $h$ small enough (this requires Assumption 3.1). Now, we consider a regular extension $\boldsymbol{n}^{*}$ of $\boldsymbol{n}$ to $\Omega$, and we take $\overline{\boldsymbol{v}}$ equal to $-\bar{q} \mathcal{I}_{h}\left(\varphi \boldsymbol{n}^{*}\right)$, which gives

$$
b(\overline{\boldsymbol{v}}, \bar{q}) \geq \frac{c_{0}}{2} \bar{q}^{2}=\frac{c_{0}}{2 \operatorname{meas}(\Omega)}\|\bar{q}\|_{L^{2}(\Omega)}^{2} \quad \text { and } \quad\|\overline{\boldsymbol{v}}\|_{\mathbb{X}} \leq c\|\bar{q}\|_{L^{2}(\Omega)} .
$$

Step 3. We conclude by using the argument due to Boland and Nicolaides [10]. We take $\boldsymbol{v}_{h}$ equal to $\tilde{\boldsymbol{v}}+\mu \overline{\boldsymbol{v}}$ for a positive constant $\mu$ and, noting that $b(\tilde{\boldsymbol{v}}, \bar{q})$ is zero, we derive from (3.4) and (3.5) that

$$
\begin{aligned}
b\left(\boldsymbol{v}_{h}, q_{h}\right) & =b(\tilde{\boldsymbol{v}}, \tilde{q})+\mu b(\overline{\boldsymbol{v}}, \bar{q})+\mu b(\overline{\boldsymbol{v}}, \tilde{q}) \\
& \geq\|\tilde{q}\|_{L^{2}(\Omega)}^{2}+\frac{\mu c_{0}}{2 \operatorname{meas}(\Omega)}\|\bar{q}\|_{L^{2}(\Omega)}^{2}-c \mu\|\tilde{q}\|_{L^{2}(\Omega)}\|\bar{q}\|_{L^{2}(\Omega)} .
\end{aligned}
$$

Using a Young's inequality thus yields

$$
b\left(\boldsymbol{v}_{h}, q_{h}\right) \geq \frac{1}{2}\|\tilde{q}\|_{L^{2}(\Omega)}^{2}+\mu\left(\frac{c_{0}}{2 \operatorname{meas}(\Omega)}-\frac{c^{2} \mu}{2}\right)\|\bar{q}\|_{L^{2}(\Omega)}^{2},
$$

whence, by taking $\mu$ equal to $\frac{c_{0}}{2 c^{2} \text { meas }(\Omega)}$ and using the orthogonality of $\tilde{q}$ and $\bar{q}$ in $L^{2}(\Omega)$, we obtain

$$
b\left(\boldsymbol{v}_{h}, q_{h}\right) \geq c^{\prime}\left\|q_{h}\right\|_{L^{2}(\Omega)}^{2} .
$$

On the other hand, we have

$$
\left\|\boldsymbol{v}_{h}\right\|_{\mathbb{X}} \leq\|\tilde{\boldsymbol{v}}\|_{\mathbb{X}}+\mu\|\overline{\boldsymbol{v}}\|_{\mathbb{X}} \leq c^{\prime \prime}\left\|q_{h}\right\|_{L^{2}(\Omega)} .
$$

This yields the desired inf-sup condition.

From now on, we suppose that Assumption 3.1 holds. On the other hand, since $\mathbb{X}_{h}$ is imbedded in $\mathbb{X}$, the ellipticity of the form $a_{1}(\cdot, \cdot)$ is a direct consequence of Lemma 2.11. So, we now state the well-posedness result.

TheOREM 3.3. Assume that the data $\boldsymbol{f}, \boldsymbol{u}_{1}, \boldsymbol{u}_{2}$, and $p_{2}$ satisfy, for a real number $\sigma>\frac{d-1}{2}$,

$$
f \in L^{2}(\Omega)^{d}, \quad \mathcal{C}\left(\boldsymbol{u}_{1}, \boldsymbol{u}_{2}\right) \in H^{\sigma}(\partial \Omega)^{d}, \quad p_{2} \in H_{00}^{\frac{1}{2}}\left(\Gamma_{2}\right) .
$$

Then, problem (3.1)-(3.2) has a unique solution $\left(\boldsymbol{u}_{h}, p_{h}\right)$. Moreover, this solution satisfies

$$
\left\|\boldsymbol{u}_{h}\right\|_{\mathbb{X}}+\left\|p_{h}\right\|_{L^{2}(\Omega)} \leq c\left(\|\boldsymbol{f}\|_{L^{2}(\Omega)^{d}}+\left\|\mathcal{C}\left(\boldsymbol{u}_{1}, \boldsymbol{u}_{2}\right)\right\|_{H^{\sigma}(\partial \Omega)^{d}}+\left\|p_{2}\right\|_{H_{00}^{\frac{1}{2}\left(\Gamma_{2}\right)}}\right) .
$$

Proof. The lifting $\boldsymbol{w}$ of the trace $\mathcal{C}\left(\boldsymbol{u}_{1}, \boldsymbol{u}_{2}\right)$ introduced in the proof of Theorem 2.5 can now be chosen in $H^{\sigma+\frac{1}{2}}(\Omega)$, at least for $\sigma$ small enough, and hence is continuous on $\bar{\Omega}$. Thus standard arguments yield

$$
\left\|\mathcal{I}_{h} \boldsymbol{w}\right\|_{H^{1}(\Omega)^{d}} \leq c\left\|\mathcal{C}\left(\boldsymbol{u}_{1}, \boldsymbol{u}_{2}\right)\right\|_{H^{\sigma}(\partial \Omega)^{d}} .
$$

Writing the problem satisfied by $\left(\boldsymbol{u}_{h}-\mathcal{I}_{h} \boldsymbol{w}, p_{h}\right)$ and combining [19, Chap. I, Cor. 4.1] with Lemmas 2.11 and 3.2 implies that problem (3.1)-(3.2) has a unique solution. Then estimate (3.7) obviously follows.

Copyright (c) by SIAM. Unauthorized reproduction of this article is prohibited. 
3.2. A priori analysis. Using the same lifting $\boldsymbol{w}$ as previously, we observe that the pair $\left(\boldsymbol{u}_{0 h}, p_{h}\right)$, with $\boldsymbol{u}_{0 h}=\boldsymbol{u}_{h}-\mathcal{I}_{h} \boldsymbol{w}$, is a solution in $\mathbb{X}_{h} \times \mathbb{M}_{h}$ of

$$
\begin{aligned}
\forall \boldsymbol{v}_{h} \in \mathbb{X}_{h}, \quad a_{1}\left(\boldsymbol{u}_{0 h}, \boldsymbol{v}_{h}\right) & +b\left(\boldsymbol{v}_{h}, p_{h}\right) \\
& =\int_{\Omega} \boldsymbol{f}(\boldsymbol{x}) \cdot \boldsymbol{v}_{h}(\boldsymbol{x}) d \boldsymbol{x}-\left\langle p_{2}, \boldsymbol{v}_{h} \cdot \boldsymbol{n}\right\rangle_{\Gamma_{2}}-a_{1}\left(\mathcal{I}_{h} \boldsymbol{w}, \boldsymbol{v}_{h}\right), \\
\forall q_{h} \in \mathbb{M}_{h}, \quad b\left(\boldsymbol{u}_{0 h}, q_{h}\right) & =-b\left(\mathcal{I}_{h} \boldsymbol{w}, q_{h}\right) .
\end{aligned}
$$

On the other hand, the pair $\left(\boldsymbol{u}_{0}, p_{0}\right)$, with $\boldsymbol{u}_{0}=\boldsymbol{u}-\boldsymbol{w}$, is a solution of the analogous continuous problem $(2.13)$ with $a(\cdot, \cdot)$ replaced by $a_{1}(\cdot, \cdot)$. So standard arguments (see [19, Chap. II, Thm. 1.1]), relying once more on Lemmas 2.11 and 3.2, yield the following version of the Strang lemma.

LEMMA 3.4. The following error estimate holds between the pairs $\left(\boldsymbol{u}_{0}, p\right)$ and $\left(\boldsymbol{u}_{0 h}, p_{h}\right):$

$$
\begin{aligned}
& \left\|\boldsymbol{u}_{0}-\boldsymbol{u}_{0 h}\right\|_{\mathbb{X}}+\left\|p-p_{h}\right\|_{L^{2}(\Omega)} \\
& \quad \leq c\left(\inf _{\boldsymbol{v}_{h} \in \mathbb{X}_{h}}\left\|\boldsymbol{u}_{0}-\boldsymbol{v}_{h}\right\|_{\mathbb{X}}+\inf _{q_{h} \in \mathbb{M}_{h}}\left\|p-q_{h}\right\|_{L^{2}(\Omega)}\right)+c^{\prime}\left\|\boldsymbol{w}-\mathcal{I}_{h} \boldsymbol{w}\right\|_{\mathbb{X}} .
\end{aligned}
$$

By using the triangle inequality

$$
\left\|\boldsymbol{u}-\boldsymbol{u}_{h}\right\|_{\mathbb{X}} \leq\left\|\boldsymbol{u}_{0}-\boldsymbol{u}_{0 h}\right\|_{\mathbb{X}}+\left\|\boldsymbol{w}-\mathcal{I}_{h} \boldsymbol{w}\right\|_{\mathbb{X}}
$$

and the approximation properties of the spaces $\mathbb{X}_{h}$ and $\mathbb{M}_{h}$ together with that of $\mathcal{I}_{h}$ (see [9, Chap. IX], for instance), we can now state the a priori estimate.

TheOrem 3.5. Assume that the data $\boldsymbol{f}, \boldsymbol{u}_{1}, \boldsymbol{u}_{2}$, and $p_{2}$ satisfy (3.6) for a real number $\sigma, \frac{d-1}{2}<\sigma \leq \frac{5}{2}$, and that the solution $(\boldsymbol{u}, p)$ of problem $(2.5)-(2.6)$ for $\varepsilon=0$ belongs to $H^{s+1}(\Omega)^{d} \times H^{s}(\Omega)$ for a real number $s, 0 \leq s \leq 2$. Then, the following a priori error estimate holds between this solution and the solution $\left(\boldsymbol{u}_{h}, p_{h}\right)$ of problem $(3.1)-(3.2)$ :

$$
\begin{aligned}
\left\|\boldsymbol{u}-\boldsymbol{u}_{h}\right\|_{\mathbb{X}} & +\left\|p-p_{h}\right\|_{L^{2}(\Omega)} \\
& \leq c h^{s}\left(\|\boldsymbol{u}\|_{H^{s+1}(\Omega)^{d}}+\|p\|_{H^{s}(\Omega)}\right)+c^{\prime} h^{\sigma-\frac{1}{2}}\left\|\mathcal{C}\left(\boldsymbol{u}_{1}, \boldsymbol{u}_{2}\right)\right\|_{H^{\sigma}(\partial \Omega)^{d}} .
\end{aligned}
$$

Clearly, this estimate is fully optimal and, when combined with (3.7), proves the convergence of the discretization for all solutions $(\boldsymbol{u}, p)$. On the other hand, for a smooth solution $(\boldsymbol{u}, p)$, the error behaves like $h^{2}$, so that the method is of order 2 .

3.3. A posteriori analysis. This analysis requires some further notation: For each element $K$ of $\mathcal{T}_{h}$,

- $\mathcal{E}_{K}$ stands for the set of edges $(d=2)$ or faces $(d=3)$ of $K$ which do not lie on $\partial \Omega$;

- $\mathcal{E}_{K}^{2}$ stands for the set of edges $(d=2)$ or faces $(d=3)$ of $K$ which lie on $\bar{\Gamma}_{2}$;

- $\omega_{K}$ denotes the union of elements of $\mathcal{T}_{h}$ that share at least an edge $(d=2)$ or a face $(d=3)$ with $K$;

- for each $e$ in $\mathcal{E}_{K},[\cdot]_{e}$ denotes the jump through $e$ (making its sign precise is not necessary in what follows);

- for each $e$ in $\mathcal{E}_{K}$ or $\mathcal{E}_{K}^{2}, h_{e}$ stands for the length $(d=2)$ or diameter $(d=3)$ of $e$.

We now intend to prove an a posteriori error estimate between the pairs $(\boldsymbol{u}, p)$ and $\left(\boldsymbol{u}_{h}, p_{h}\right)$, solutions of problems (2.5)-(2.6) and (3.1)-(3.2), respectively. The first 
residual equation reads, for all $\boldsymbol{v}$ in $\mathbb{X}$ and $\boldsymbol{v}_{h}$ in $\mathbb{X}_{h}$,

$$
\begin{array}{r}
a_{1}\left(\boldsymbol{u}-\boldsymbol{u}_{h}, \boldsymbol{v}\right)+b\left(\boldsymbol{v}, p-p_{h}\right)=\int_{\Omega} \boldsymbol{f}(\boldsymbol{x}) \cdot\left(\boldsymbol{v}-\boldsymbol{v}_{h}\right)(\boldsymbol{x}) d \boldsymbol{x}-\left\langle p_{2},\left(\boldsymbol{v}-\boldsymbol{v}_{h}\right) \cdot \boldsymbol{n}\right\rangle_{\Gamma_{2}} \\
-a_{1}\left(\boldsymbol{u}_{h}, \boldsymbol{v}-\boldsymbol{v}_{h}\right)-b\left(\boldsymbol{v}-\boldsymbol{v}_{h}, p_{h}\right) .
\end{array}
$$

When integrating by parts on each element $K$ of $\mathcal{T}_{h}$, this gives

$$
\begin{aligned}
& a_{1}\left(\boldsymbol{u}-\boldsymbol{u}_{h}, \boldsymbol{v}\right)+b\left(\boldsymbol{v}, p-p_{h}\right) \\
& \begin{aligned}
\sum_{K \in \mathcal{T}_{h}} & \left(\int_{K}\left(\boldsymbol{f}-\nu \operatorname{curl}\left(\operatorname{curl} \boldsymbol{u}_{h}\right)+\nu \operatorname{grad}\left(\operatorname{div} \boldsymbol{u}_{h}\right)-\operatorname{grad} p_{h}\right)(\boldsymbol{x}) \cdot\left(\boldsymbol{v}-\boldsymbol{v}_{h}\right)(\boldsymbol{x}) d \boldsymbol{x}\right. \\
& +\frac{1}{2} \sum_{e \in \mathcal{E}_{K}} \int_{e} \nu\left(\left[\operatorname{curl} \boldsymbol{u}_{h}\right]_{e}(\tau) \cdot\left(\boldsymbol{v}-\boldsymbol{v}_{h}\right) \times \boldsymbol{n}(\tau)-\left[\operatorname{div} \boldsymbol{u}_{h}\right]_{e}(\tau)\left(\boldsymbol{v}-\boldsymbol{v}_{h}\right) \cdot \boldsymbol{n}(\tau)\right) d \tau
\end{aligned} \\
& \left.\quad+\sum_{e \in \mathcal{E}_{K}^{2}} \int_{e}\left(p_{2}-\nu \operatorname{div} \boldsymbol{u}_{h}-p_{h}\right)(\tau)\left(\boldsymbol{v}-\boldsymbol{v}_{h}\right) \cdot \boldsymbol{n}(\tau) d \tau\right) .
\end{aligned}
$$

Fortunately, the second residual equation is much simpler. It reads, for any $q$ in $L^{2}(\Omega)$,

$$
b\left(\boldsymbol{u}-\boldsymbol{u}_{h}, q\right)=-b\left(\boldsymbol{u}_{h}, q\right) .
$$

To go further, we introduce an approximation $\boldsymbol{f}_{h}$ of $\boldsymbol{f}$ in $\mathbb{M}_{h}^{d}$, for instance, and an approximation $p_{2 h}$ of $p_{2}$ which is continuous and affine on each edge $(d=2)$ or face $(d=3)$ contained in $\Gamma_{2}$. Thanks to (3.11) and (3.12), we are now in a position to define the error indicators. They read, for each $K$ in $\mathcal{T}_{h}$,

$$
\begin{aligned}
\eta_{K}=h_{K}\left\|\boldsymbol{f}_{h}-\nu \operatorname{curl}\left(\operatorname{curl} \boldsymbol{u}_{h}\right)-\operatorname{grad} p_{h}\right\|_{L^{2}(K)^{d}}+\left\|\operatorname{div} \boldsymbol{u}_{h}\right\|_{L^{2}(K)} \\
+\sum_{e \in \mathcal{E}_{K}} h_{e}^{\frac{1}{2}}\left\|\left[\operatorname{curl} \boldsymbol{u}_{h}\right]_{e}\right\|_{L^{2}(e)} \frac{d(d-1)}{2}+\sum_{e \in \mathcal{E}_{K}^{2}} h_{e}^{\frac{1}{2}}\left\|p_{2 h}-p_{h}\right\|_{L^{2}(e)} .
\end{aligned}
$$

These indicators are very easy to compute since they involve only polynomials of low degree.

Remark 3.6. The term due to the jump of $\operatorname{curl} \boldsymbol{u}_{h}$ in the indicator $\eta_{K}$ defined by (3.13) may be simplified to

$$
\sum_{e \in \mathcal{E}_{K}} h_{e}^{1 / 2}\left\|\left[\partial_{n} \boldsymbol{u}_{h t}\right]_{e}\right\|_{L^{2}(e) \frac{d(d-1)}{2}},
$$

where $\partial_{n}$ denotes the normal derivative and $\boldsymbol{u}_{h t}$ are the tangential components of the velocity $\boldsymbol{u}_{h}$ on $e$. This occurs because in (3.11) we have

$$
\left[\operatorname{curl} \boldsymbol{u}_{h} \times \boldsymbol{n}\right]_{e}=\left[\left(\operatorname{curl} \boldsymbol{u}_{h} \times \boldsymbol{n}\right)_{t}\right]_{e}=\left[\partial_{n} \boldsymbol{u}_{h \tau}\right]_{e} \quad \text { on } e,
$$

where the second equality holds because the tangential derivatives do not jump across $e$. Similarly the term $\boldsymbol{f}_{h}-\nu \operatorname{curl}\left(\operatorname{curl} \boldsymbol{u}_{h}\right)-\operatorname{grad} p_{h}$ can be replaced by $\boldsymbol{f}_{h}+\nu \Delta \boldsymbol{u}_{h}-$ $\operatorname{grad} p_{h}$. With these modifications, it may be easier to see that these indicators are of residual type (which means that, when suppressing the indices $h$, they vanish). However, the expression for the curl term in (3.13) leads to an easier computation in practice. 
We are now in a position to state the a posteriori error estimate. For this, we introduce a neighborhood $\mathcal{V}$ of the re-entrant corners and edges in $\Gamma_{2}$ and set

$$
s_{K}= \begin{cases}\frac{1}{2} & \text { if } K \subset \mathcal{V} \\ 0 & \text { otherwise. }\end{cases}
$$

THEOREM 3.7. The following a posteriori error estimate holds between the solution $(\boldsymbol{u}, p)$ of problem (2.5)-(2.6) for $\varepsilon=0$ and the solution $\left(\boldsymbol{u}_{h}, p_{h}\right)$ of problem $(3.1)-(3.2)$ :

$$
\left\|\boldsymbol{u}-\boldsymbol{u}_{h}\right\|_{\mathbb{X}}+\left\|p-p_{h}\right\|_{L^{2}(\Omega)} \leq c\left(\sum_{K \in \mathcal{T}_{h}} h_{K}^{-2 s_{K}} \eta_{K}^{2}\right)^{\frac{1}{2}}+\varepsilon_{h},
$$

where the quantity $\varepsilon_{h}$ is defined by

$$
\begin{gathered}
\varepsilon_{h}=\left(\sum_{K \in \mathcal{T}_{h}}\left(h_{K}^{2\left(1-s_{K}\right)}\left\|\boldsymbol{f}-\boldsymbol{f}_{h}\right\|_{L^{2}(K)^{d}}^{2}+\sum_{e \in \mathcal{E}_{K}^{2}} h_{e}^{1-2 s_{K}}\left\|p_{2}-p_{2 h}\right\|_{L^{2}(e)}^{2}\right)\right)^{\frac{1}{2}} \\
+\left\|\mathcal{C}\left(\boldsymbol{u}_{1}, \boldsymbol{u}_{2}\right)-\mathcal{I}_{h} \mathcal{C}\left(\boldsymbol{u}_{1}, \boldsymbol{u}_{2}\right)\right\|_{H^{\frac{1}{2}}(\partial \Omega)^{d}} .
\end{gathered}
$$

Proof. We observe from (3.11) and (3.12) that the pair $\left(\boldsymbol{u}-\boldsymbol{u}_{h}, p-p_{h}\right)$ is a solution of problem (2.5)-(2.6) with data equal to the right-hand side $\mathcal{R}$ of (3.11), the quantity $\mathcal{C}\left(\boldsymbol{u}_{1}, \boldsymbol{u}_{2}\right)-\mathcal{I}_{h} \mathcal{C}\left(\boldsymbol{u}_{1}, \boldsymbol{u}_{2}\right)$, and the right-hand side of (3.12). Thus, estimate (3.15) will follow by applying estimate (2.12) to this new problem. The quantity $\mathcal{C}\left(\boldsymbol{u}_{1}, \boldsymbol{u}_{2}\right)-\mathcal{I}_{h} \mathcal{C}\left(\boldsymbol{u}_{1}, \boldsymbol{u}_{2}\right)$ and the right-hand side of (3.12) are obviously bounded. To evaluate $\mathcal{R}$, we apply a Cauchy-Schwarz inequality, take $\boldsymbol{v}_{h}$ equal to the image of $\boldsymbol{v}$ by a Clément-type regularization operator $\mathcal{R}_{h}$ with values in $\mathbb{X}_{h}$, and recall from $[9$, sect. IX.3] or [33, Prop. 3.33] that, for any $s \geq \frac{1}{2}$ and for any $e$ in $\mathcal{E}_{K}$ or in $\mathcal{E}_{K}^{2}$,

$$
\left\|\boldsymbol{v}-\mathcal{R}_{h} \boldsymbol{v}\right\|_{L^{2}(K)^{d}} \leq c h_{K}^{s}\|\boldsymbol{v}\|_{H^{s}\left(\omega_{K}\right)}, \quad\left\|\boldsymbol{v}-\mathcal{R}_{h} \boldsymbol{v}\right\|_{L^{2}(e)^{d}} \leq c h_{e}^{s-\frac{1}{2}}\|\boldsymbol{v}\|_{H^{s}\left(\omega_{K}\right)} .
$$

To conclude, we note from Remark 2.1 that functions $\boldsymbol{v}$ in $\mathbb{X}$ belong to $H^{1}(\Omega \backslash \mathcal{V})$ but only to $H^{\frac{1}{2}}(\mathcal{V})$, and we get rid of the further terms involving $\operatorname{div} \boldsymbol{u}_{h}$ by using the inverse inequalities [9, Chap. VII, Prop. 4.1], [33, Prop. 3.37],

$$
\begin{gathered}
h_{K}\left\|\operatorname{grad}\left(\operatorname{div} \boldsymbol{u}_{h}\right)\right\|_{L^{2}(K)^{d}} \leq c\left\|\operatorname{div} \boldsymbol{u}_{h}\right\|_{L^{2}(K)}, \\
h_{e}^{\frac{1}{2}}\left\|\operatorname{div} \boldsymbol{u}_{h}\right\|_{L^{2}(e)} \leq c\left\|\operatorname{div} \boldsymbol{u}_{h}\right\|_{L^{2}(K)} .
\end{gathered}
$$

All this yields the desired estimate.

Estimate (3.15) is optimal when the domain $\Omega$ is convex in a neighborhood of $\Gamma_{2}$. Moreover, the lack of optimality in the general case is local, limited to $\mathcal{V}$, and exactly the same was noted in [8, Prop. 5.3] for another type of mixed boundary conditions. We now prove a local upper bound for the indicators. For each $K$ in $\mathcal{T}_{h}$, we denote by $\|\cdot\|_{\mathbb{X}(K)}$ the restriction of the norm $\|\cdot\|_{\mathbb{X}}$ to $K$, with obvious extension to $\omega_{K}$.

Proposition 3.8. Each indicator $\eta_{K}, K \in \mathcal{T}_{h}$, defined in (3.13) satisfies

$$
\eta_{K} \leq c\left(\left\|\boldsymbol{u}-\boldsymbol{u}_{h}\right\|_{\mathbb{X}\left(\omega_{K}\right)}+\left\|p-p_{h}\right\|_{L^{2}\left(\omega_{K}\right)}+\varepsilon_{K}\right),
$$

where the quantity $\varepsilon_{K}$ is defined by

$$
\varepsilon_{K}=h_{K}\left\|\boldsymbol{f}-\boldsymbol{f}_{h}\right\|_{L^{2}\left(\omega_{K}\right)^{d}}+\sum_{e \in \mathcal{E}_{K}^{2}} h_{e}^{\frac{1}{2}}\left\|p_{2}-p_{2 h}\right\|_{L^{2}(e)} .
$$

Copyright $@$ by SIAM. Unauthorized reproduction of this article is prohibited. 
Proof. Since the arguments are fully standard, we only give an abridged version of the proof. We successively bound the four terms in $\eta_{K}$.

Step 1. We set

$$
\boldsymbol{v}_{K}= \begin{cases}\left(\boldsymbol{f}_{h}-\nu \operatorname{curl}\left(\operatorname{curl} \boldsymbol{u}_{h}\right)+\nu \operatorname{grad}\left(\operatorname{div} \boldsymbol{u}_{h}\right)-\operatorname{grad} p_{h}\right) \psi_{K} & \text { on } K, \\ \mathbf{0} & \text { elsewhere }\end{cases}
$$

where $\psi_{K}$ is the bubble function on $K$ (equal to the product of the barycentric coordinates associated with the vertices of $K)$. Next, we take $\boldsymbol{v}$ equal to $\boldsymbol{v}_{K}$, and $\boldsymbol{v}_{h}$ equal to zero in (3.11). Standard inverse inequalities (see [33, Prop. 3.37]) lead to

$$
\begin{aligned}
h_{K} \| \boldsymbol{f}_{h}-\nu \operatorname{curl}\left(\operatorname{curl} \boldsymbol{u}_{h}\right) & +\nu \operatorname{grad}\left(\operatorname{div} \boldsymbol{u}_{h}\right)-\operatorname{grad} p_{h} \|_{L^{2}(K)^{d}} \\
& \leq c\left(\left\|\boldsymbol{u}-\boldsymbol{u}_{h}\right\|_{\mathbb{X}(K)}+\left\|p-p_{h}\right\|_{L^{2}(K)}+h_{K}\left\|\boldsymbol{f}-\boldsymbol{f}_{h}\right\|_{L^{2}(K)^{d}}\right),
\end{aligned}
$$

or, equivalently, by using (3.17),

$$
\begin{aligned}
& h_{K}\left\|\boldsymbol{f}_{h}-\nu \operatorname{curl}\left(\operatorname{curl} \boldsymbol{u}_{h}\right)-\operatorname{grad} p_{h}\right\|_{L^{2}(K)^{d}} \\
& \quad \leq c\left(\left\|\boldsymbol{u}-\boldsymbol{u}_{h}\right\|_{\mathbb{X}(K)}+\left\|p-p_{h}\right\|_{L^{2}(K)}+h_{K}\left\|\boldsymbol{f}-\boldsymbol{f}_{h}\right\|_{L^{2}(K)^{d}}\right)+c^{\prime}\left\|\operatorname{div} \boldsymbol{u}_{h}\right\|_{L^{2}(K)} .
\end{aligned}
$$

Step 2. We set

$$
q_{K}= \begin{cases}\left(\operatorname{div} \boldsymbol{u}_{h}\right) \chi_{K} & \text { on } K \\ 0 & \text { elsewhere }\end{cases}
$$

where $\chi_{K}$ is the characteristic function of $K$. Taking $q$ equal to $q_{K}$ in (3.12) gives

$$
\left\|\operatorname{div} \boldsymbol{u}_{h}\right\|_{L^{2}(K)} \leq\left\|\boldsymbol{u}-\boldsymbol{u}_{h}\right\|_{\mathbb{X}(K)} .
$$

Combining (3.20) and (3.21) gives the estimate for the first two terms in $\eta_{K}$.

Step 3 . For each edge $(d=2)$ or face $(d=3) e$ of $K$, we consider a lifting operator $\mathcal{L}_{e, K}$ that maps polynomials of fixed degree on $e$ vanishing on $\partial e$ into polynomials vanishing on $\partial K \backslash e$ and is constructed from a fixed lifting operator on the reference triangle or tetrahedron. If an element $e$ of $\mathcal{E}_{K}$ is shared by two elements $K$ and $K^{\prime}$, we set

$$
\boldsymbol{v}_{e}= \begin{cases}\mathcal{L}_{e, \kappa}\left(\left[\operatorname{curl} \boldsymbol{u}_{h}\right]_{e} \psi_{e}\right) & \text { on } \kappa \in\left\{K, K^{\prime}\right\} \\ \mathbf{0} & \text { elsewhere }\end{cases}
$$

where $\psi_{e}$ is now the bubble function on $e$. We take $\boldsymbol{v}$ equal to $\tilde{\boldsymbol{v}}_{e}$, and $\boldsymbol{v}_{h}$ equal to zero in (3.11), where $\tilde{\boldsymbol{v}}_{e}$ is such that

$$
\tilde{\boldsymbol{v}}_{e} \times \boldsymbol{n}=\boldsymbol{v}_{e} \times \boldsymbol{n} \text { and } \quad \tilde{\boldsymbol{v}}_{e} \cdot \boldsymbol{n}=0 \quad \text { on } e .
$$

Standard arguments [33, Prop. 3.37], combined with (3.20) and (3.21), yield

$$
\begin{aligned}
& h_{e}^{\frac{1}{2}}\left\|\left[\operatorname{curl} \boldsymbol{u}_{h}\right]_{e}\right\|_{L^{2}(e)^{\frac{d(d-1)}{2}}} \\
& \quad \leq c\left(\left\|\boldsymbol{u}-\boldsymbol{u}_{h}\right\|_{\mathbb{X}\left(K \cup K^{\prime}\right)}+\left\|p-p_{h}\right\|_{L^{2}\left(K \cup K^{\prime}\right)}+h_{K}\left\|\boldsymbol{f}-\boldsymbol{f}_{h}\right\|_{L^{2}\left(K \cup K^{\prime}\right)^{d}}\right) .
\end{aligned}
$$

Step 4. For each $e$ in $\mathcal{E}_{K}^{2}$, we set

$$
\boldsymbol{v}_{e}= \begin{cases}\mathcal{L}_{e, K}\left(\left(p_{2 h}-p_{h}\right) \boldsymbol{n} \psi_{e}\right) & \text { on } K \\ \mathbf{0} & \text { elsewhere }\end{cases}
$$

Copyright (c) by SIAM. Unauthorized reproduction of this article is prohibited. 
We finally take $\boldsymbol{v}$ equal to $\boldsymbol{v}_{e}$, and $\boldsymbol{v}_{h}$ equal to zero in (3.11), which gives

$$
\begin{aligned}
& h_{e}^{\frac{1}{2}}\left\|p_{2 h}-p_{h}\right\|_{L^{2}(e)} \\
& \quad \leq c\left(\left\|\boldsymbol{u}-\boldsymbol{u}_{h}\right\|_{\mathbb{X}(K)}+\left\|p-p_{h}\right\|_{L^{2}(K)}+h_{K}\left\|\boldsymbol{f}-\boldsymbol{f}_{h}\right\|_{L^{2}(K)^{d}}+h_{e}^{\frac{1}{2}}\left\|p_{2}-p_{2 h}\right\|_{L^{2}(e)}\right) .
\end{aligned}
$$

Owing to the definition (3.19) of $\varepsilon_{K}$, estimate (3.18) follows from (3.20) to (3.23).

Estimate (3.18) is fully optimal. Moreover, it is local, which proves the efficiency of our indicators for mesh adaptivity.

4. Discretization of the Navier-Stokes equations. We use here all the notation of section 3 . We write the nonlinear discrete problem. Next, we prove simultaneously the existence of a solution and the a priori error estimate by following the approach due to Brezzi, Rappaz, and Raviart [12]. We conclude by extending the results of a posteriori analysis to the nonlinear case.

4.1. The discrete problem. As previously, the discrete problem associated with problem (2.6)-(2.14) (for $\varepsilon=1$ ) is constructed by the Galerkin method. It reads: Find $\left(\boldsymbol{u}_{h}, p_{h}\right)$ in $\mathbb{X}_{h} \times \mathbb{M}_{h}$ such that

$$
\begin{aligned}
\forall \boldsymbol{v}_{h} \in \mathbb{X}_{h}, \quad a_{1}\left(\boldsymbol{u}_{h}, \boldsymbol{v}_{h}\right)+ & N\left(\boldsymbol{u}_{h}, \boldsymbol{u}_{h}, \boldsymbol{v}_{h}\right)+b\left(\boldsymbol{v}_{h}, p_{h}\right) \\
& =\int_{\Omega} \boldsymbol{f}(\boldsymbol{x}) \cdot \boldsymbol{v}_{h}(\boldsymbol{x}) d \boldsymbol{x}-\left\langle p_{2}, \boldsymbol{v}_{h} \cdot \boldsymbol{n}\right\rangle_{\Gamma_{2}}, \\
\forall q_{h} \in \mathbb{M}_{h}, \quad b\left(\boldsymbol{u}_{h}, q_{h}\right)=0 . &
\end{aligned}
$$

The existence of a solution for this problem can be proved by the same arguments as in section 2.3. However, we prefer to perform its numerical analysis directly.

4.2. A priori analysis. We now introduce a different notation. Let $\mathcal{S}$ denote the operator which associates with $\left(\boldsymbol{f}, p_{2}\right)$ in $L^{2}(\Omega)^{d} \times H_{00}^{\frac{1}{2}}\left(\Gamma_{2}\right)$ the solution $(\boldsymbol{u}, p)$ of problem (2.6)-(2.14) with $\varepsilon=0$, namely of the Stokes problem with zero boundary conditions on the velocity. Then, problem (2.6)-(2.14) with $\varepsilon=1$ can equivalently be written as

$$
\mathcal{F}(\boldsymbol{u}, p)=(\boldsymbol{u}, p)-\mathcal{S}\left(\boldsymbol{g}(\boldsymbol{u}), p_{2}\right)=0
$$

where the function $\boldsymbol{g}$ is defined by duality

$$
\langle\boldsymbol{g}(\boldsymbol{u}), \boldsymbol{v}\rangle=\int_{\Omega} \boldsymbol{f}(\boldsymbol{x}) \cdot \boldsymbol{v}(\boldsymbol{x}) d \boldsymbol{x}-N(\boldsymbol{u}, \boldsymbol{u}, \boldsymbol{v}) .
$$

Similarly, let $\mathcal{S}_{h}$ denote the operator which associates with $\left(\boldsymbol{f}, p_{2}\right)$ in $L^{2}(\Omega)^{d} \times H_{00}^{\frac{1}{2}}\left(\Gamma_{2}\right)$ the solution $\left(\boldsymbol{u}_{h}, p_{h}\right)$ of problem (3.1)-(3.2) with zero boundary conditions $\boldsymbol{u}_{1}=\boldsymbol{u}_{2}=$ $\mathbf{0}$ on the velocity, more precisely of the following problem: Find $\left(\boldsymbol{u}_{h}, p_{h}\right)$ in $\mathbb{X}_{h} \times \mathbb{M}_{h}$ such that

$$
\begin{array}{ll}
\forall \boldsymbol{v}_{h} \in \mathbb{X}_{h}, \quad a_{1}\left(\boldsymbol{u}_{h}, \boldsymbol{v}_{h}\right)+b\left(\boldsymbol{v}_{h}, p_{h}\right)=\int_{\Omega} \boldsymbol{f}(\boldsymbol{x}) \cdot \boldsymbol{v}_{h}(\boldsymbol{x}) d \boldsymbol{x}-\left\langle p_{2}, \boldsymbol{v}_{h} \cdot \boldsymbol{n}\right\rangle_{\Gamma_{2}}, \\
\forall q_{h} \in \mathbb{M}_{h}, \quad b\left(\boldsymbol{u}_{h}, q_{h}\right)=0 .
\end{array}
$$

Then, problem (4.1) can equivalently be written

$$
\mathcal{F}_{h}\left(\boldsymbol{u}_{h}, p_{h}\right)=\left(\boldsymbol{u}_{h}, p_{h}\right)-\mathcal{S}_{h}\left(\boldsymbol{g}\left(\boldsymbol{u}_{h}\right), p_{2}\right)=0 .
$$

Copyright (c) by SIAM. Unauthorized reproduction of this article is prohibited. 
Denoting by $\mathcal{Z}$ the space $\mathbb{X} \times L^{2}(\Omega)$, we recall from Theorems 3.3 and 3.5 the main properties of the operators $\mathcal{S}_{h}$ : its stability,

$$
\left\|\mathcal{S}_{h}\left(\boldsymbol{f}, p_{2}\right)\right\|_{\mathcal{Z}} \leq c\left(\|\boldsymbol{f}\|_{L^{2}(\Omega)^{d}}+\left\|p_{2}\right\|_{H_{00}^{\frac{1}{2}}\left(\Gamma_{2}\right)}\right),
$$

and the error estimate, for a smooth enough solution $\mathcal{S}\left(\boldsymbol{f}, p_{2}\right)$ and $s \leq 2$,

$$
\left\|\left(\mathcal{S}-\mathcal{S}_{h}\right)\left(\boldsymbol{f}, p_{2}\right)\right\|_{\mathcal{Z}} \leq c h^{s}\left\|\mathcal{S}\left(\boldsymbol{f}, p_{2}\right)\right\|_{H^{s+1}(\Omega)^{d} \times H^{s}(\Omega)} .
$$

All of this gives the convergence property, for any $\left(\boldsymbol{f}, p_{2}\right)$ in $L^{2}(\Omega)^{d} \times H_{00}^{\frac{1}{2}}\left(\Gamma_{2}\right)$,

$$
\lim _{h \rightarrow 0}\left\|\left(\mathcal{S}-\mathcal{S}_{h}\right)\left(\boldsymbol{f}, p_{2}\right)\right\|_{\mathcal{Z}}=0 .
$$

Due to Lemma 3.4, this convergence easily extends to data $\left(\boldsymbol{f}, p_{2}\right)$ in $\mathbb{X}^{\prime} \times H_{00}^{\frac{1}{2}}\left(\Gamma_{2}\right)$, where $\mathbb{X}^{\prime}$ stands for the dual space of $\mathbb{X}$.

We are thus in a position to prove some preliminary results. As usual, they require a further assumption.

ASSUMPTION 4.1. We consider a solution $(\boldsymbol{u}, p)$ of problem (2.6)-(2.14) with $\varepsilon=1$, which

(i) belongs to $H^{s+1}(\Omega)^{d} \times H^{s}(\Omega)$ for a real number $s, 0<s \leq 2$, and

(ii) is such that $D \mathcal{F}(\boldsymbol{u}, p)$ is an isomorphism of $\mathcal{Z}$ (where $D$ denotes the differential operator).

This assumption is much weaker than the uniqueness of the solution established in Theorem 2.10, since part (ii) only implies the local uniqueness of the solution. We denote by $\mathcal{L}(\mathcal{Z})$ the space of endomorphisms of $\mathcal{Z}$. Owing to properties (4.6)-(4.8), the proof of the next lemma is fully standard, and so we skip it.

Lemma 4.2. If Assumptions 2.8 and 4.1 hold, then

(i) there exists an $h_{0}>0$ such that, for $h \leq h_{0}, D \mathcal{F}_{h}(\boldsymbol{u}, p)$ is an isomorphism of $\mathcal{Z}$ and the norm of its inverse is bounded independently of $h$;

(ii) there exist a neighborhood $\mathcal{V}$ of $(\boldsymbol{u}, p)$ in $\mathcal{Z}$ and a constant $\Lambda>0$ such that the mapping $D \mathcal{F}_{h}$ satisfies the Lipschitz property

$$
\forall(\boldsymbol{v}, q) \in \mathcal{V}, \quad\left\|D \mathcal{F}_{h}(\boldsymbol{u}, p)-D \mathcal{F}_{h}(\boldsymbol{v}, q)\right\|_{\mathcal{L}(\mathcal{Z})} \leq \Lambda\|(\boldsymbol{u}, p)-(\boldsymbol{v}, q)\|_{\mathcal{Z}} ;
$$

(iii) the following bound holds,

$$
\left\|\mathcal{F}_{h}(\boldsymbol{u}, p)\right\|_{\mathcal{Z}} \leq c(\boldsymbol{u}, p) h^{s},
$$

for a constant $c(\boldsymbol{u}, p)$ depending only on the regularity of $(\boldsymbol{u}, p)$.

Owing to Lemma 4.2, all the assumptions needed for [12, Thm. 1] (see also [19, Chap. IV, Thm. 3.1]) are satisfied. So applying this theorem leads to the main result of this section.

THEOREM 4.3. If Assumptions 2.8 and 4.1 hold, there exist an $h_{*}>0$ and a neighborhood $\mathcal{V}_{*}$ of $(\boldsymbol{u}, p)$ in $\mathcal{Z}$ such that, for $h \leq h_{*}$, problem (4.1) has a unique solution $\left(\boldsymbol{u}_{h}, p_{h}\right)$ in $\mathcal{V}_{*}$. Moreover, the following a priori error estimate is satisfied:

$$
\left\|\boldsymbol{u}-\boldsymbol{u}_{h}\right\|_{\mathbb{X}}+\left\|p-p_{h}\right\|_{L^{2}(\Omega)} \leq c(\boldsymbol{u}, p) h^{s}
$$

for a constant $c(\boldsymbol{u}, p)$ depending only on $(\boldsymbol{u}, p)$.

Copyright $@$ by SIAM. Unauthorized reproduction of this article is prohibited. 
4.3. A posteriori analysis. The second residual equation (3.12) is the same as in the linear case, but unfortunately the first residual equation is a little more complex. After integration by parts on each $K$, it reads, for all $\boldsymbol{v}$ in $\mathbb{X}$ and $\boldsymbol{v}_{h}$ in $\mathbb{X}_{h}$,

$$
a_{1}\left(\boldsymbol{u}-\boldsymbol{u}_{h}, \boldsymbol{v}\right)+N(\boldsymbol{u}, \boldsymbol{u}, \boldsymbol{v})-N\left(\boldsymbol{u}_{h}, \boldsymbol{u}_{h}, \boldsymbol{v}\right)+b\left(\boldsymbol{v}, p-p_{h}\right)=\mathcal{R}_{1}+\mathcal{R}_{2}+\mathcal{R}_{3},
$$

where

$$
\begin{aligned}
& \mathcal{R}_{1}=\sum_{K \in \mathcal{T}_{h}} \int_{K}\left(\boldsymbol{f}-\nu \operatorname{curl}\left(\operatorname{curl} \boldsymbol{u}_{h}\right)+\nu \operatorname{grad}\left(\operatorname{div} \boldsymbol{u}_{h}\right)\right. \\
& \left.-\left(\boldsymbol{u}_{h} \cdot \nabla\right) \boldsymbol{u}_{h}-\operatorname{grad} p_{h}\right)(\boldsymbol{x}) \cdot\left(\boldsymbol{v}-\boldsymbol{v}_{h}\right)(\boldsymbol{x}) d \boldsymbol{x}, \\
& \mathcal{R}_{2}=\frac{1}{2} \sum_{K \in \mathcal{T}_{h}} \sum_{e \in \mathcal{E}_{K}} \int_{e} \nu\left(\left[\operatorname{curl} \boldsymbol{u}_{h}\right]_{e}(\tau) \cdot\left(\boldsymbol{v}-\boldsymbol{v}_{h}\right) \times \boldsymbol{n}(\tau)\right. \\
& \left.-\left[\operatorname{div} \boldsymbol{u}_{h}\right]_{e}(\tau)\left(\boldsymbol{v}-\boldsymbol{v}_{h}\right) \cdot \boldsymbol{n}(\tau)\right) d \tau, \\
& \mathcal{R}_{3}=\sum_{K \in \mathcal{T}_{h}} \sum_{e \in \mathcal{E}_{K}^{2}} \int_{e}\left(p_{2}-\nu \operatorname{div} \boldsymbol{u}_{h}-p_{h}-\frac{1}{2}\left|\boldsymbol{u}_{h}\right|^{2}\right)(\tau)\left(\boldsymbol{v}-\boldsymbol{v}_{h}\right) \cdot \boldsymbol{n}(\tau) d \tau \text {. }
\end{aligned}
$$

This leads to the definition of the error indicators: For each $K$ in $\mathcal{T}_{h}$, with the same notation as previously,

$$
\eta_{K}=h_{K}\left\|\boldsymbol{f}_{h}-\nu \operatorname{curl}\left(\operatorname{curl} \boldsymbol{u}_{h}\right)-\left(\boldsymbol{u}_{h} \cdot \nabla\right) \boldsymbol{u}_{h}-\operatorname{grad} p_{h}\right\|_{L^{2}(K)^{d}}+\left\|\operatorname{div} \boldsymbol{u}_{h}\right\|_{L^{2}(K)}
$$

$$
+\sum_{e \in \mathcal{E}_{K}} h_{e}^{\frac{1}{2}}\left\|\left[\operatorname{curl} \boldsymbol{u}_{h}\right]_{e}\right\|_{L^{2}(e)} \frac{d(d-1)}{2}+\sum_{e \in \mathcal{E}_{K}^{2}} h_{e}^{\frac{1}{2}}\left\|p_{2 h}-p_{h}-\frac{1}{2}\left|\boldsymbol{u}_{h}\right|^{2}\right\|_{L^{2}(e)} .
$$

Even if the nonlinear terms add polynomials of higher degree, these indicators are still easy to compute.

In order to apply the theorem due to Pousin and Rappaz [29], we need a further notation: Let $\mathcal{S}^{*}$ denote the operator which associates with $\left(\boldsymbol{f}, \chi, p_{2}\right)$ in $L^{2}(\Omega)^{d} \times$ $L^{2}(\Omega) \times H_{00}^{\frac{1}{2}}\left(\Gamma_{2}\right)$ the solution $(\boldsymbol{u}, p)$ in $\mathbb{X} \times L^{2}(\Omega)$ of the problem

$$
\begin{aligned}
& \forall \boldsymbol{v} \in \mathbb{X}, \quad a(\boldsymbol{u}, \boldsymbol{v})+b(\boldsymbol{v}, p)=\int_{\Omega} \boldsymbol{f}(\boldsymbol{x}) \cdot \boldsymbol{v}(\boldsymbol{x}) d \boldsymbol{x}-\left\langle p_{2}, \boldsymbol{v} \cdot \boldsymbol{n}\right\rangle_{\Gamma_{2}}, \\
& \forall q \in L^{2}(\Omega), \quad b(\boldsymbol{u}, q)=\int_{\Omega} \chi(\boldsymbol{x}) q(\boldsymbol{x}) d \boldsymbol{x} .
\end{aligned}
$$

(The introduction of this more complex operator is due to the fact that the right-hand side of (3.12) is not zero.) Then, problem (2.6)-(2.14) with $\varepsilon=1$ can equivalently be written

$$
\mathcal{F}^{*}(\boldsymbol{u}, p)=(\boldsymbol{u}, p)-\mathcal{S}^{*}\left(\boldsymbol{g}(\boldsymbol{u}), 0, p_{2}\right)=0 .
$$

We are now in a position to prove the a posteriori error estimate.

THEOREM 4.4. For any solution $(\boldsymbol{u}, p)$ of problem (2.6)-(2.14) with $\varepsilon=1$ such that $D \mathcal{F}^{*}(\boldsymbol{u}, p)$ is an isomorphism of $\mathcal{Z}$, there exists a neighborhood $\mathcal{V}_{* *}$ of $(\boldsymbol{u}, p)$ in $\mathcal{Z}$ such that the following a posteriori error estimate is satisfied for any solution $\left(\boldsymbol{u}_{h}, p_{h}\right)$ of problem (4.1) in $\mathcal{V}_{* *}$ :

$$
\left\|\boldsymbol{u}-\boldsymbol{u}_{h}\right\|_{\mathbb{X}}+\left\|p-p_{h}\right\|_{L^{2}(\Omega)} \leq c\left(\sum_{K \in \mathcal{T}_{h}} h_{K}^{-2 s_{K}} \eta_{K}^{2}\right)^{\frac{1}{2}}+\varepsilon_{h},
$$

Copyright $@$ by SIAM. Unauthorized reproduction of this article is prohibited. 
where the parameter $s_{K}$ is defined in (3.14) and the quantity $\varepsilon_{h}$ in (3.16).

Proof. The same arguments as for Lemma 4.2 imply that $D \mathcal{F}^{*}$ is Lipschitzcontinuous in a neighborhood of $(\boldsymbol{u}, p)$. So we apply the theorem due to Pousin and Rappaz [29] (see also [33, Prop. 5.1]): Any solution of problem (4.1) in this neighborhood satisfies

$$
\left\|\boldsymbol{u}-\boldsymbol{u}_{h}\right\|_{\mathbb{X}}+\left\|p-p_{h}\right\|_{L^{2}(\Omega)} \leq\left\|\mathcal{F}^{*}\left(\boldsymbol{u}_{h}, p_{h}\right)\right\|_{\mathcal{Z}},
$$

whence, due to (4.16),

$$
\left\|\boldsymbol{u}-\boldsymbol{u}_{h}\right\|_{\mathbb{X}}+\left\|p-p_{h}\right\|_{L^{2}(\Omega)} \leq\left\|\mathcal{F}^{*}(\boldsymbol{u}, p)-\mathcal{F}^{*}\left(\boldsymbol{u}_{h}, p_{h}\right)\right\|_{\mathcal{Z}} .
$$

Due to the stability property of $\mathcal{S}^{*}$, estimating the right-hand side of this equation relies on (4.12) and (3.12) and is performed by the same arguments as were used for Theorem 3.7.

To prove the converse estimate, we observe that

$$
N(\boldsymbol{u}, \boldsymbol{u}, \boldsymbol{v})-N\left(\boldsymbol{u}_{h}, \boldsymbol{u}_{h}, \boldsymbol{v}\right)=N\left(\boldsymbol{u}-\boldsymbol{u}_{h}, \boldsymbol{u}, \boldsymbol{v}\right)+N\left(\boldsymbol{u}_{h}, \boldsymbol{u}-\boldsymbol{u}_{h}, \boldsymbol{v}\right) .
$$

So, when working with bounded $\boldsymbol{u}$ and $\boldsymbol{u}_{h}$, proving the next proposition relies on exactly the same arguments as for Proposition 3.8, now applied to (4.12) and (3.12).

Proposition 4.5. For any solution $\left(\boldsymbol{u}_{h}, p_{h}\right)$ of problem (4.1) in a neighborhood of $(\boldsymbol{u}, p)$, each indicator $\eta_{K}, K \in \mathcal{T}_{h}$, defined in (4.14) satisfies

$$
\eta_{K} \leq c\left(\left\|\boldsymbol{u}-\boldsymbol{u}_{h}\right\|_{\mathbb{X}\left(\omega_{K}\right)}+\left\|p-p_{h}\right\|_{L^{2}\left(\omega_{K}\right)}+\varepsilon_{K}\right),
$$

where the quantity $\varepsilon_{K}$ is defined in (3.19).

5. Numerical experiments. The next computations are performed on the code due to Hecht and Pironneau, called FreeFem++; see [20]. We start from a coarse initial mesh and perform adaptivity following the next criterion: The diameter of each new triangle containing an element $K$ or contained in an element $K$ of the old triangulation is proportional to $h_{K} \frac{\bar{\eta}}{\eta_{K}}$, where $\bar{\eta}$ is the mean value of the $\eta_{K}$.

We work with the Navier-Stokes equations for the data $\boldsymbol{f}=\mathbf{0}$. So we use the following iterative algorithm to treat the nonlinear term: Assuming that the solution of the time-dependent problem with time-independent data converges to the solution $\left(\boldsymbol{u}_{h}, p_{h}\right)$ of our problem, we solve the time-dependent problem via an implicit Euler scheme where the nonlinear term is treated in a semiexplicit way. On each mesh, we iterate this algorithm until its convergence, i.e., until the difference between two consecutive solutions becomes smaller than a fixed tolerance.

First, we consider the two-dimensional domain made of two pipes; see Figure 1(left). Let $P_{1}$ be the horizontal pipe and $P_{2}$ the vertical one. The boundary $\Gamma_{2}$ is made of the vertical edge of $P_{1}$ (on the left) and of the two horizontal edges of $P_{2}$, while $\Gamma_{1}$ is equal to $\partial \Omega \backslash \bar{\Gamma}_{2}$.

We take the viscosity $\nu$ equal to 0.025 . The geometry and the data are similar to those suggested in [4, sect. 3.4.1]; in particular, the data on the velocity are zero as in (2.14), and the data on the pressure are a constant on each connected component of $\Gamma_{2}$ (see Remark 2.6 for the justification of that).

In the first test case, the constants on the two edges of $P_{2}$ are equal, so that, since the viscosity $\nu$ is large enough, the flow remains symmetric. More precisely and with obvious notation, these constants are given by $c_{1}=0, c_{2-}=c_{2+}=-2$. Figure 1 (right) presents a zoom of the final adapted mesh near the re-entrant corners. Figure 2 illustrates the velocity $\boldsymbol{u}_{h}$ and the pressure $p_{h}$ on this last mesh. 

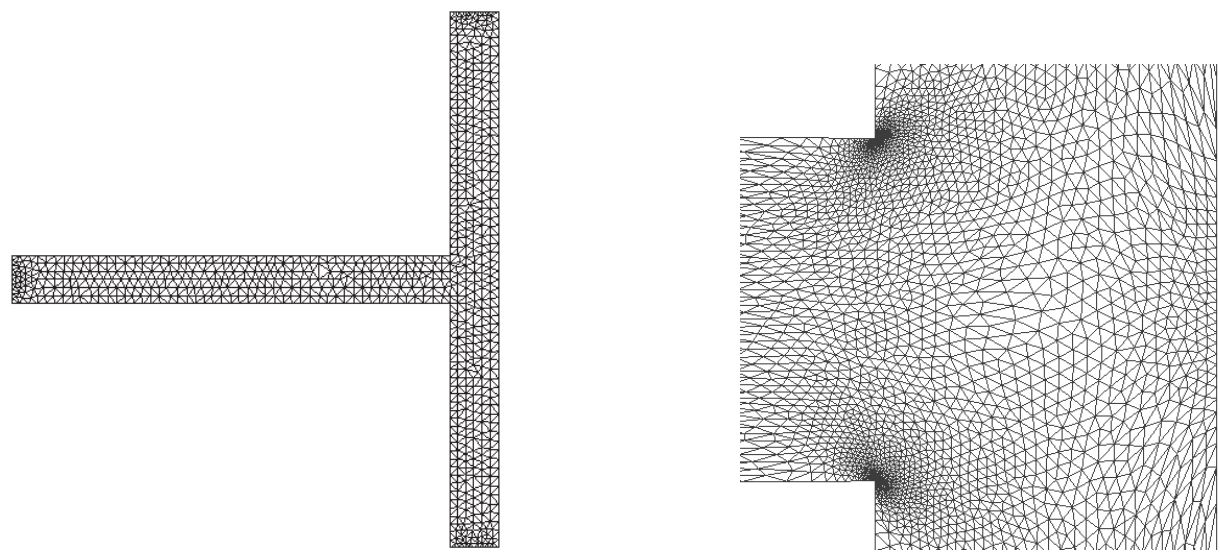

FIG. 1. Left: The domain $\Omega$ and its initial mesh. Right: Zoom of the adapted mesh.

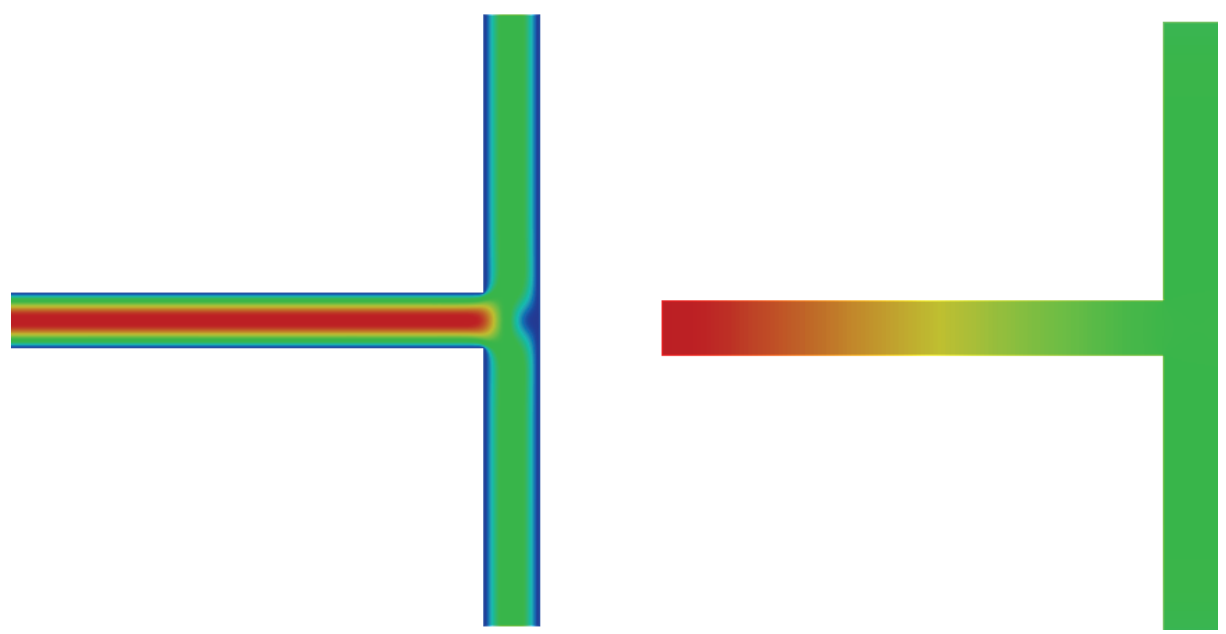

FIG. 2. The discrete velocity $\boldsymbol{u}_{h}$ (left) and pressure $p_{h}$ (right).

In the second test case, the data are the same, but the constants on the two edges of $P_{2}$ are rather different, given by $c_{1}=0, c_{2-}=-4, c_{2+}=-2$. Figure 3 presents a zoom of the final adapted mesh. Figure 4 illustrates the velocity $\boldsymbol{u}_{h}$ and the pressure $p_{h}$ for these new values. All these results are in good agreement with [4, Figs. 3.2 and 3.3].

Next, we study the case of a flow behind a spherical obstacle, as illustrated in Figure 5 (left). The viscosity is taken equal to $\frac{1}{55}$, and, with $\Gamma_{2}$ equal to the union of the two vertical edges of $\partial \Omega$, the pressure is given equal to 5 in the left edge and to 3 on the right edge. The final adapted mesh is presented in Figure 5(right), and the corresponding velocity in Figure 6. The existence of the Von Karman vortex street is undeniable. There also, these results are very similar to those in [4, Fig. 3.4].

We conclude with the case of a three-dimensional channel flow, which is one of the most popular test problems for the investigation of wall bounded turbulent flows. This flow is well fitted to testing our pressure boundary conditions for the NavierStokes equations, as it is driven by a pressure jump between the inflow and outflow 


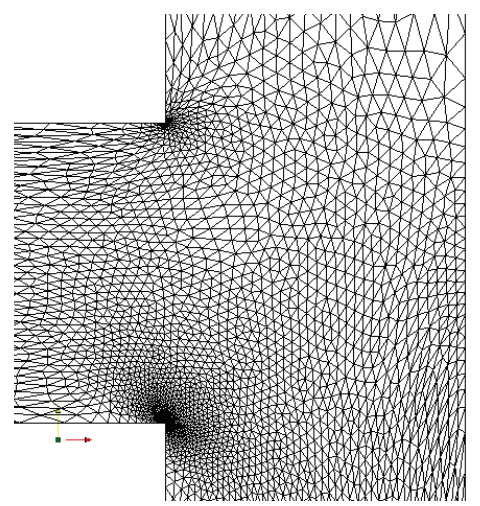

FIG. 3. Zoom of the final adapted mesh.

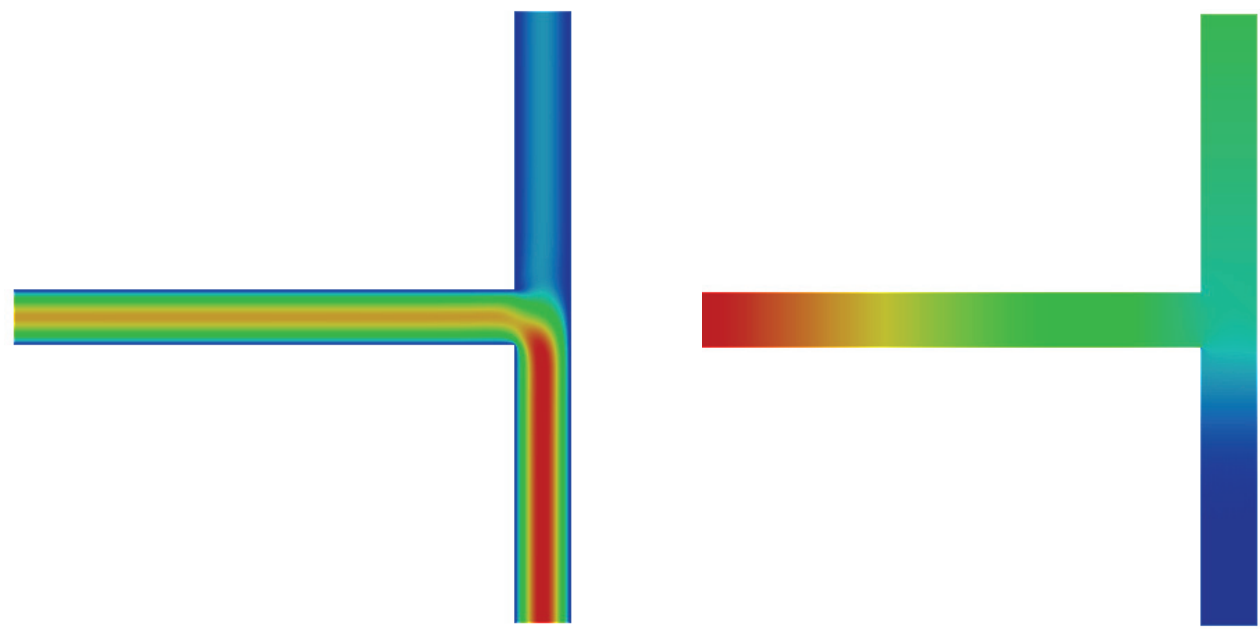

FIG. 4. The discrete velocity $\boldsymbol{u}_{h}$ (left) and pressure $p_{h}$ (right).

boundaries. In the usual formulation of Navier-Stokes equations this pressure jump is modeled by means of a forcing term.

The characteristic parameter of the turbulent channel flow is the friction Reynolds

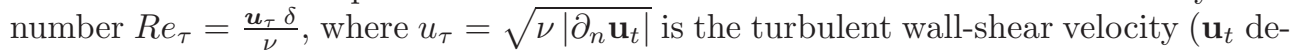
notes the tangential velocity at the wall) and $\delta$ is the channel half-width. We consider the computational domain $\Omega=\left(0, L_{1}\right) \times(-\delta, \delta) \times\left(0, L_{3}\right)$, with $\delta=1$ (wall-normal direction), $L_{1}=2 \pi$ (streamwise direction), and $L_{3}=(4 / 3) \pi$ (spanwise direction). The boundary conditions are periodic in both the streamwise and spanwise directions. The viscosity is $\nu=1 / 180$. The Reynolds number based on a unit friction velocity reachable at a steady state is then $R e_{\tau}=180$.

In the standard formulation of Navier-Stokes equations, the flow is driven by a constant forcing $\boldsymbol{f}=\left(f_{p}, 0,0\right)=(1,0,0)$, which models an imposed pressure gradient in the streamwise direction. The specific choice of a unit value for $f_{p}$ aims at obtaining a unit value for $u_{\tau}$ in the statistically steady state, subject to the relation $u_{\tau}=\sqrt{f_{p} h}$ (cf. [18]). This corresponds to a pressure jump $p_{\text {out }}-p_{\text {in }}=L_{1}$.

We use the projection-based VMS (Variational MultiScale) turbulence model de- 

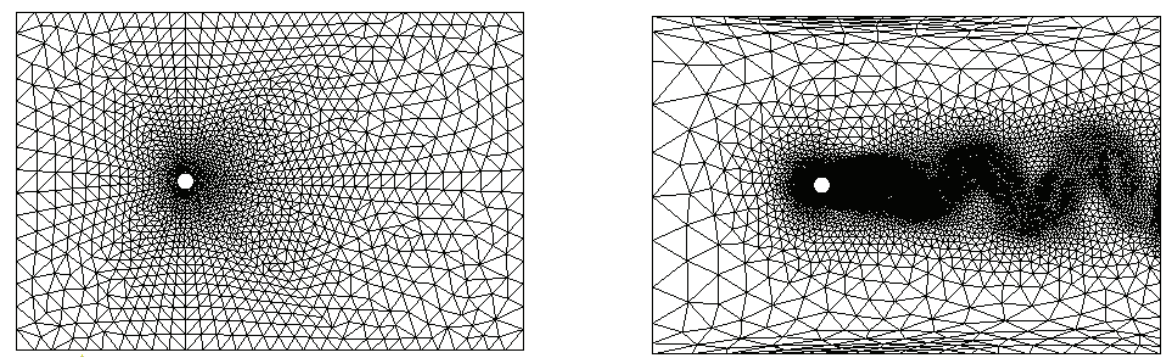

FIG. 5. The initial (left) and final (right) adapted meshes.

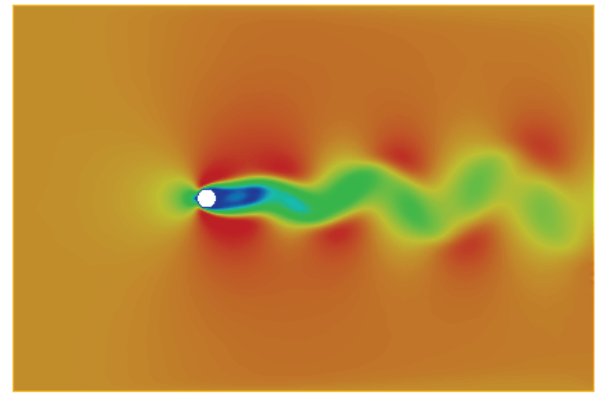

FIG. 6. The discrete velocity $\boldsymbol{u}_{h}$.

scribed in [13, Chap. 11], which for brevity we do not detail here. In this model the subgrid flow is modeled by means of Smagorkinsky-like eddy diffusion term with projection structure. To impose the boundary conditions on the pressure, we just reformulate the Navier-Stokes equations as in (2.6) and keep the same subgrid modeling terms as in the VMS model. We impose no-slip boundary conditions on the upper and lower walls. We compare second-order statistics as measure of turbulence intensities, for three models: The original VMS method (Method 1) with forcing term, the present method with Dirichlet pressure boundary conditions (Method 2), both with $32 \times 32 \times 32$ degrees of freedom, and a direct numerical simulation (DNS) of Moser, Kim, and Mansour [26] with forcing term, obtained with $128 \times 128 \times 128$ degrees of freedom. Figures 7,8 , and 9 display the normalized (by $u_{\tau}$ ) root-mean-square (r.m.s.) values of velocity fluctuations in wall coordinates,

$$
y^{+}=\frac{u_{\tau}}{\nu} y,
$$

at the upper half-width of the channel. The errors with respect to the DNS simulation of Methods 1 and 2 are comparable for all three fluctuations. The errors for the streamwise velocity fluctuations are smaller for Method 1, while those for the crosswise velocity fluctuations are smaller for Method 2. All these results are in good agreement with the computations performed by Rubino; see [13, Chap. 11]. We thus obtain similar results with our formulation imposing pressure jump conditions, as we might expect.

6. Conclusions. Fluid flows in pipes and channels usually are driven by pressure jumps between inlet and outlet boundaries. The study of such boundary conditions is thus of high applied interest. We have performed in this work the a priori and a posteriori error analysis of linear and nonlinear models of fluid flow including these kinds 


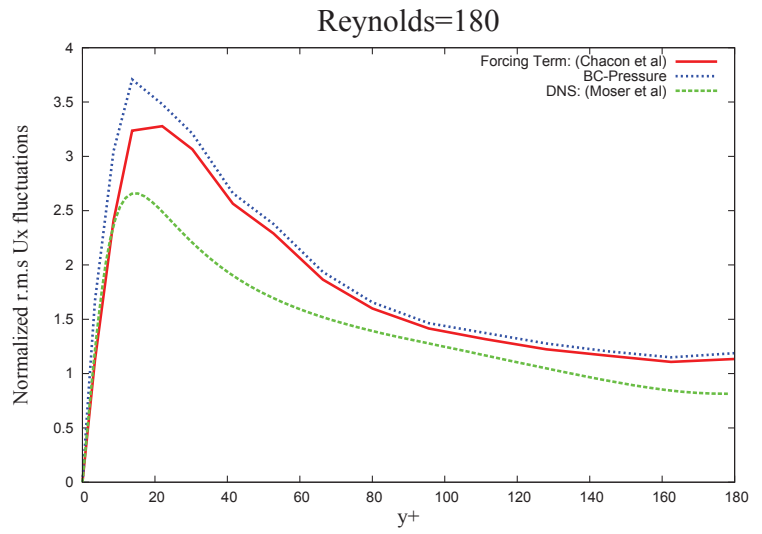

FIG. 7. Normalized r.m.s. $U_{x}$ velocity fluctuations profiles in wall coordinates $y^{+}$.

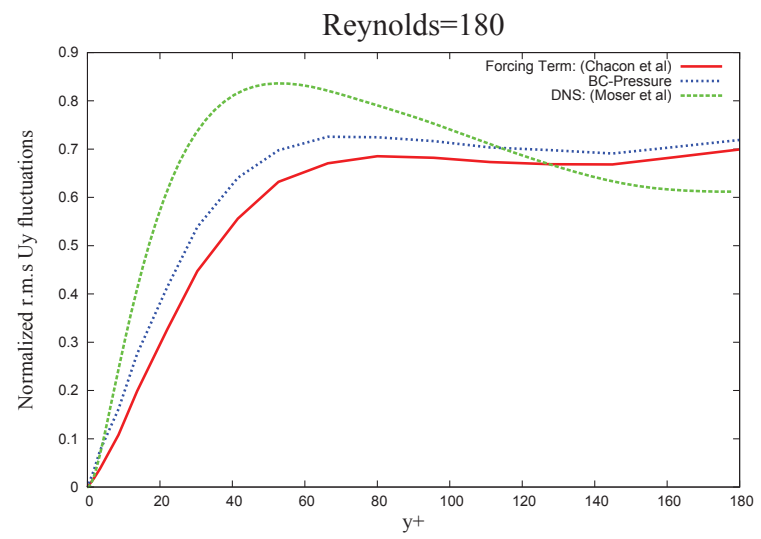

FIG. 8. Normalized r.m.s. $U_{y}$ velocity fluctuations profiles in wall coordinates $y^{+}$.

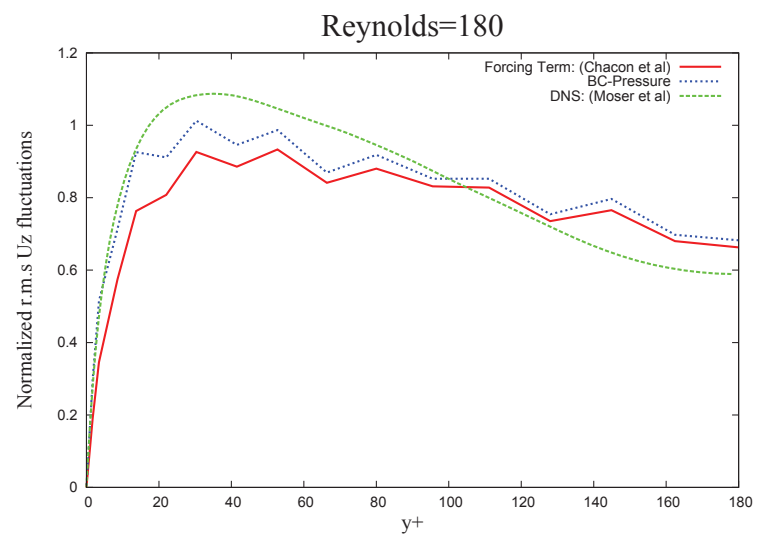

FIG. 9. Normalized r.m.s. $U_{z}$ velocity fluctuations profiles in wall coordinates $y^{+}$.

Copyright (c) by SIAM. Unauthorized reproduction of this article is prohibited. 
of conditions for the pressure, which complements several previous works on the same subject. We have addressed pressure boundary conditions with some smoothness across boundary corners, in order to be able to give a weak sense to our formulations. The smoothness of the boundary also plays a crucial role in using compactness arguments to treat the convection term in the Navier-Stokes equations.

We have applied the a posteriori error analysis to building a grid refinement strategy, which we have tested in some relevant pipe and channel flows. We have verified the a priori error analysis for smooth functions and tested the convergence for boundary conditions with low regularity, not covered by our analysis. Our results are highly satisfying in all cases.

We conclude that our formulation of Navier-Stokes equations with pressure boundary conditions is appropriate for performing an adaptive grid strategy for pressure boundary conditions with $L^{2}$ regularity on the boundary. We believe that the analysis of less smooth pressure boundary conditions is possible with a weaker definition of the solution that allows us to use smoother test functions, maybe by transposition.

Acknowledgment. The authors are very grateful to Samuele Rubino for interesting discussions and comparison of their computations.

\section{REFERENCES}

[1] R. A. Adams and J. Fournier, Sobolev Spaces, Academic Press, New York, 2003.

[2] C. Amrouche, C. Bernardi, M. Dauge, and V. Girault, Vector potentials in threedimensional nonsmooth domains, Math. Methods Appl. Sci., 21 (1998), pp. 823-864.

[3] C. Amrouche and N. E. H. Seloula, $L^{p}$-theory for the Navier-Stokes equations with pressure boundary conditions, Discrete Contin. Dyn. Syst. Ser. S, 6 (2013), pp. 1113-1137.

[4] C. Bègue, C. Conca, F. Murat, and O. Pironneau, Les équations de Stokes et de NavierStokes avec des conditions aux limites sur la pression, in Nonlinear Partial Differential Equations and Their Applications, Collège de France Seminar IX, H. Brezis and J.-L.Lions, eds., Pitman, Boston, 1988, pp. 179-264.

[5] C. Bernardi, C. Canuto, And Y. Maday, Spectral approximations of the Stokes equations with boundary conditions on the pressure, SIAM J. Numer. Anal., 28 (1991), pp. 333-362.

[6] C. Bernardi, T. Chacón Rebollo, R. Lewandowski, and F. Murat, a model for two coupled turbulent fluids. Part I: Analysis of the system, Collège de France Seminar XIV, D. Cioranescu and J.-L. Lions, eds., North-Holland, Amsterdam, 2002, pp. 69-102.

[7] C. Bernardi and N. Chorfi, Spectral discretization of the vorticity, velocity, and pressure formulation of the Stokes problem, SIAM J. Numer. Anal., 44 (2006), pp. 826-850.

[8] C. Bernardi, F. Hecht, and R. Verfürth, A finite element discretization of the threedimensional Navier-Stokes equations with mixed boundary conditions, Math. Model. Numer. Anal., 43 (2009), pp. 1185-1201.

[9] C. Bernardi, Y. Maday, AND F. RApetTi, Discrétisations variationnelles de problèmes aux limites elliptiques, Collection "Mathématiques et Applications" 45, Springer-Verlag, Berlin, 2004.

[10] J. M. Boland And R. A. Nicolaides, Stability of finite elements under divergence constraints, SIAM J. Numer. Anal., 20 (1983), pp. 722-731.

[11] H. Brezis, Analyse fonctionnelle, Théorie et applications, Masson, Paris, 1987.

[12] F. Brezzi, J. Rappaz, and P.-A. Raviart, Finite dimensional approximation of nonlinear problems, Part I: Branches of nonsingular solutions, Numer. Math., 36 (1980), pp. 1-25.

[13] T. Chacón Rebollo and R. Lewandowski, Mathematical and Numerical Foundations of Turbulence Models and Applications, Birkhäuser, Cambridge, MA, 2014.

[14] C. Conca, F. Murat, and O. Pironneau, The Stokes and Navier-Stokes equations with boundary conditions involving the pressure, Japan. J. Math., 20 (1994), pp. 279-318.

[15] M. Costabel, A remark on the regularity of solutions of Maxwell's equations on Lipschitz domains, Math. Methods Appl. Sci., 12 (1990), pp. 365-368.

[16] F. Dubois, Vorticity-velocity-pressure formulation for the Stokes problem, Math. Methods Appl. Sci., 25 (2002), pp. 1091-1119. 
[17] F. Dubois, M. Salaün, And S. Salmon, Vorticity-velocity-pressure and stream functionvorticity formulations for the Stokes problem, J. Math. Pures Appl., 82 (2003), pp. 13951451.

[18] T. Dubois, F. Jauberteau, and R. Temam, Dynamic Multilevel Methods and the Numerical Simulation of Turbulence, Cambridge University Press, Cambridge, UK, 1999.

[19] V. Girault And P.-A. Raviart, Finite Element Methods for Navier-Stokes Equations, Theory and Algorithms, Springer-Verlag, New York, Berlin, 1986.

[20] F. Hecht, New development in FreeFem++, J. Numer. Math., 20 (2012), pp. 251-266.

[21] P. Hood And C. TAYlor, A numerical solution of the Navier-Stokes equations using the finite element technique, Comput. \& Fluids., 1 (1973), pp. 73-100.

[22] S. M. Hosseini And J. J. Feng, Pressure boundary conditions for computing incompressible flows with SPH, J. Comput. Phys., 230 (2011), pp. 7473-7487.

[23] H. Johnston And J.-G. Liu, Finite difference schemes for incompressible flow based on local pressure boundary conditions, J. Comput. Phys., 180 (2002), pp. 120-154.

[24] J.-L. Lions and E. Magenes, Problèmes aux limites non homogènes et applications, Vol. I, Dunod, Paris, 1968.

[25] S. MARuŠIĆ, On the Navier-Stokes system with pressure boundary condition, Ann. Univ. Ferrara Sez. VII Sci. Mat., 53 (2007), pp. 319-331.

[26] R. Moser, J. Kim, And N. N. Mansour, Direct numerical simulation of turbulent channel flow up to $R e_{\tau}=590$, Phys. Fluids., 11 (1999), pp. 943-945.

[27] N. A. Petersson, Stability of pressure boundary conditions for Stokes and Navier-Stokes equations, J. Comput. Phys., 172 (2001), pp. 4-70.

[28] O. Pironneau, Conditions aux limites sur la pression pour les équations de Stokes et de Navier-Stokes, C. R. Acad. Sci. Paris Sér. I Math., 303 (1986), pp. 403-406.

[29] J. Pousin And J. RAppaz, Consistency, stability, a priori and a posteriori errors for PetrovGalerkin methods applied to nonlinear problems, Numer. Math., 69 (1994), pp. 213-231.

[30] S. Salmon, Développement numérique de la formulation tourbillon-vitesse-pression pour le problème de Stokes, Ph.D. thesis, Université Pierre et Marie Curie, Paris, France, 1999.

[31] R. L. Sani, J. Shen, O. Pironneau, and P. M. Gresho, Pressure boundary condition for the time-dependent incompressible Navier-Stokes equations, Internat. J. Numer. Methods Fluids., 50 (2006), pp. 673-682.

[32] R. Temam, Navier-Stokes Equations. Theory and Numerical Analysis, Stud. Math. Appl. 2, North-Holland, Amsterdam, 1977.

[33] R. Verfürth, A Posteriori Error Estimation Techniques for Finite Element Methods, Oxford University Press, London, 2013.

Copyright (c) by SIAM. Unauthorized reproduction of this article is prohibited. 